\title{
Image Extrapolation for the Time Discrete Metamorphosis Model: Existence and Applications*
}

\author{
Alexander Effland ${ }^{\dagger}$, Martin Rumpf ${ }^{\dagger}$, and Florian Schäfer ${ }^{\ddagger}$
}

\begin{abstract}
The space of images can be equipped with a Riemannian metric measuring both the cost of transport of image intensities and the variation of image intensities along motion lines. The resulting metamorphosis model was introduced and analyzed in [M. I. Miller and L. Younes, Int. J. Comput. Vis., 41 (2001), pp. 61-84; A. Trouvé and L. Younes, Found. Comput. Math., 5 (2005), pp. 173-198], and a variational time discretization for the geodesic interpolation was proposed in [B. Berkels, A. Effland, and M. Rumpf, SIAM J. Imaging Sci., 8 (2015), pp. 1457-1488]. In this paper, this time discrete model is expanded and an image extrapolation via a discretization of the geometric exponential map is consistently derived for the variational time discretization. For a given weakly differentiable initial image and an initial image variation, the exponential map allows one to compute a discrete geodesic extrapolation path in the space of images. It is shown that a time step of this shooting method can be formulated in the associated deformations only. For sufficiently small time steps, local existence and uniqueness are proved using a suitable fixed point formulation and the implicit function theorem. A spatial Galerkin discretization with cubic splines on coarse meshes for the deformations and piecewise bilinear finite elements on fine meshes for the image intensities are used to derive a fully practical algorithm. Different applications underline the efficiency and stability of the proposed approach.
\end{abstract}

Key words. shape space, metamorphosis, variational time discretization, image extrapolation

AMS subject classifications. 65D18, 37L65, 49M25, 65L20

DOI. $10.1137 / 17 \mathrm{M} 1129544$

1. Introduction. Riemannian geometry has influenced imaging and computer vision tremendously in the past decades. In particular, many methods in image processing have benefited from concepts emerging from Riemannian geometry like geodesic curves, the logarithm, the exponential map, and parallel transport. For example, when considering the space of images as an infinite-dimensional Riemannian manifold, the exponential map of an input image w.r.t. an initial variation corresponds to an image extrapolation in the direction of this infinitesimal variation. In particular, the large deformation diffeomorphic metric mapping (LDDMM) framework proved to be a powerful tool underpinned by the rigorous mathemati-

\footnotetext{
${ }^{*}$ Received by the editors May 9, 2017; accepted for publication (in revised form) January 10, 2018; published electronically March 15, 2018. A preliminary version of this paper appeared in Proceedings of the International Conference on Scale Space and Variational Methods in Computer Vision, Lecture Notes in Comput. Sci. 10302, Springer, Cham, 2017, pp. 473-485.

http://www.siam.org/journals/siims/11-1/M112954.html

Funding: The research of the first and second authors was supported by the Hausdorff Center for Mathematics and the Collaborative Research Center 1060 funded by the German Research Foundation.

${ }^{\dagger}$ Institute for Numerical Simulation, University of Bonn, Bonn 53115, Germany (alexander.effland@ins.uni-bonn. de, martin.rumpf@ins.uni-bonn.de).

${ }^{\ddagger}$ Department of Computing and Mathematical Sciences, California Institute of Technology, Pasadena, CA 91125 (florian.schaefer@caltech.edu).
} 
cal theory of diffeomorphic flows. In fact, Dupuis, Grenander, and Miller [8] showed that the resulting flow is actually a flow of diffeomorphisms. Trouvé [29, 30] exploited Lie group methods to construct a distance in the space of deformations. In [18], Joshi and Miller applied this framework to (inexact and exact) landmark matching. Beg et al. [4] studied EulerLagrange equations for minimizing vector fields in the LDDMM framework and proposed an efficient algorithm incorporating a gradient descent scheme and a semi-Lagrangian method to integrate the velocity fields. Miller, Trouvé, and Younes [23] proved the conservation of the initial momentum in Lagrangian coordinates associated with a geodesic in the LDDMM framework, which allows for the stable computation of geodesic curves. Younes [36] used Jacobi fields in the flow of diffeomorphisms approach to derive gradient descent methods for the path energy. In [15], Hart, Zach, and Niethammer exploited the optimal control perspective to the LDDMM model with the motion field as the underlying control. Vialard et. al. [33, 34] studied methods from optimal control theory to accurately estimate this initial momentum and to relate it to the Hamiltonian formulation of the geodesic flow. Furthermore, they used the associated Karcher mean to compute intrinsic means of medical images. Vialard and Santambrogio investigated in [35] the flow of diffeomorphisms approach for images that are represented as functions of bounded variation. In particular, they were able to rigorously derive an Euler-Lagrange equation for the formulation with a matching energy. Lorenzi and Pennec [20] applied the LDDMM framework to compute geodesics and parallel transport using Lie group methods.

The metamorphosis model [24, 32] generalizes the flow of diffeomorphisms approach, allowing for intensity variations along transport paths, and associates a corresponding cost functional with these variations. In [31], Trouvé and Younes rigorously analyzed the local geometry of the resulting Riemannian manifold and proved the existence of geodesic curves for square-integrable images and the (local) existence as well as the uniqueness of solutions of the initial value problem for the geodesic equation in the case of images with square-integrable weak derivatives. Holm, Trouvé, and Younes [16] studied a Lagrangian formulation for the metamorphosis model and proved existence for both the boundary value problem and the initial value problem in the case of measure-valued images. Hong et al. [17] proposed a metamorphic regression model and developed a shooting method to reliably recover initial momenta.

A comprehensive overview of most of the aforementioned topics is given in the book by Younes [37]. For a historic account, we additionally refer the reader to [22].

In [6], a variational time discretization of the metamorphosis model based on a sequence of simple, elastic image matching problems was introduced and $\Gamma$-convergence to the time continuous metamorphosis model was proven. Furthermore, using a finite element discretization in space a robust algorithm was derived. Here we study not the boundary value problem of geodesic interpolation but the initial value problem of geodesic extrapolation. Exploiting de Casteljau's algorithm, this approach could also be used to compute discrete Riemannian Bézier curves in the space of images [10]. In [5], the geodesic interpolation proposed in [6] was employed to analyze the temporal evolution of a macular degeneration for medical images acquired with an optical coherence tomography device, where an efficient GPU implementation is used to speed up the registration subproblems.

In this paper, we focus on the discretization of the geometric exponential map-which 
we will denote for simplicity discrete exponential map - associated with the time discrete metamorphosis model. The Euler-Lagrange equations of the discrete path energy proposed in [6] give rise to a set of equations which characterize time steps of a discrete initial value problem for a given initial image and a given initial image variation. We study this time stepping problem both analytically and numerically. We will prove existence and uniqueness of solutions of the single time step problem, which is guaranteed to generate time discrete geodesics in the sense of the time discrete variational approach. A straightforward treatment, for instance via a Newton scheme, would lead to higher order derivatives of image functions concatenated with diffeomorphic deformations, which are both theoretically and numerically very difficult to treat. We show how to avoid these difficulties using a proper transformation of the defining Euler-Lagrange equations and reduce the number of unknowns. W.r.t. the existence, we apply a fixed point argument based on Banach's fixed point theorem for images bounded in $H^{1}$ and an initial variation, which is supposed to be small in $L^{2}$. The uniqueness proof is based on an implicit function theorem argument for initial variations, which are small in $H^{1}$. Finally, the numerical algorithm picks up the fixed point approach for another variant of the Euler-Lagrange equations.

Compared to the previous proceedings paper [9], in which the discrete exponential map in the context of the time discrete metamorphosis model and the numerical optimization algorithm were introduced, we present in this paper a comprehensive derivation of the method and formulate and prove the local existence as well as the local uniqueness of the discrete exponential map. Furthermore, two additional numerical applications are given.

Our variational discretization is related to the discrete mechanics approach originating from the work of Marsden, Ortiz, Veselov, West, and coworkers (see [21, 26] and the references therein). In this approach, the principal scheme is as follows. One considers a quadrature approximation of the action map $y \mapsto \int_{(k-1) \tau}^{k \tau} L(y(t), \dot{y}(t)) \mathrm{d} t$ on a time interval $[(k-1) \tau, k \tau]$ for $k \in\{1, \ldots, K\}$ for a time step size $\tau=\frac{1}{K}$, where $L$ denotes the associated Lagrangian. Now, one iteratively minimizes this discretized action functional for given (approximate) initial data $(y((k-1) \tau), \dot{y}((k-1) \tau))$ at time $(k-1) \tau$ to obtain the approximation $y(k \tau), \dot{y}(k \tau)$ at time $k \tau$. One main advantage is the preservation of momentum maps along certain Lagrangian flows, which is implied by the symplectic structure of the flows and Noether's theorem. Different from the general discrete mechanics approach and the underlying quadrature of the action map, the starting point of the variational time discretization ansatz introduced in [28], which we pick up here, is an approximation of the squared Riemannian distance via a pairwise energy $\mathcal{W}$ in the sense that $\left|\mathcal{W}[y, \tilde{y}]-\operatorname{dist}^{2}(y, \tilde{y})\right| \leq C \operatorname{dist}^{3}(y, \tilde{y})$ for all points $y, \tilde{y}$ in the manifold. This approximation is in general not based on a numerical quadrature of the path energy. In fact, we are particularly interested in functionals $\mathcal{W}$ which are easier to evaluate than the original path energy on a single time interval. The functional $\mathcal{W}$ considered here in the metamorphosis model is indeed significantly simpler than a direct quadrature approximation of the metamorphosis path energy. Our approximation is justified by the $\Gamma$-convergence result proven in [6]. Nevertheless, the underlying variational nature of the time discretization is fairly similar to the one in the discrete mechanics approach. In particular, as we will emphasize later, the time discrete exponential shooting leads to a discrete geodesic which is a minimizer of the associated discrete path energy, as long as this minimizer is unique. Let us mention that 
many results in the discrete mechanics framework are applicable only in finite dimensions, whereas our approximation tool applies to the space of square-integrable images equipped with the nonlinear Riemannian structure of the metamorphosis model without any space discretization.

The paper is organized as follows: In section 2, we briefly recall the metamorphosis model in the time continuous and time discrete settings, respectively. Departing from the EulerLagrange equations of a time discrete geodesic, a single time step of the discrete exponential map is derived in section 3. Then the discrete geodesic shooting relies on the iterative application of the one-step extrapolation. In section 4, local existence and local uniqueness of this discrete exponential map are proven based on a suitable combination of the implicit function theorem and Banach's fixed point theorem. The fixed point formulation is also used in section 5 to derive an efficient and stable algorithm. Finally, numerical results for different applications are presented in section 6 .

We use standard notation for Lebesgue and Sobolev spaces on the image domain $\Omega$, i.e., $L^{p}(\Omega)$ and $H^{m}(\Omega)=W^{m, 2}(\Omega)$. The associated norms are denoted by $\|\cdot\|_{L^{p}(\Omega)}$ and $\|\cdot\|_{H^{m}(\Omega)}$, respectively, and the seminorm in $H^{m}(\Omega)$ is given by $|\cdot|_{H^{m}(\Omega)}$. For any $f, g \in H^{m}(\Omega), m \geq 1$, we set

$$
D^{m} f \cdot D^{m} g=\sum_{i_{1}, \ldots, i_{m}=1}^{n} \frac{\partial^{m} f}{\partial_{x_{i_{1}}} \cdots \partial_{x_{i_{m}}}} \cdot \frac{\partial^{m} g}{\partial_{x_{i_{1}}} \cdots \partial_{x_{i_{m}}}}, \quad\left|D^{m} f\right|=\left(D^{m} f \cdot D^{m} f\right)^{\frac{1}{2}} .
$$

Then the Sobolev (semi-)norm is defined as

$$
|f|_{H^{m}(\Omega)}=\left\|D^{m} f\right\|_{L^{2}(\Omega)}, \quad\|f\|_{H^{m}(\Omega)}=\left(\sum_{j=0}^{m}|f|_{H^{j}(\Omega)}^{2}\right)^{\frac{1}{2}} .
$$

Furthermore, $H_{0}^{m}(\Omega)$ is the closure of $C^{\infty}(\Omega)$ functions with compact support w.r.t. the Sobolev norm $\|\cdot\|_{H^{m}(\Omega)}$, and its dual is denoted by $H^{-m}(\Omega)$.

The polyharmonic operator is inductively defined by $\Delta^{m} f:=\Delta\left(\Delta^{m-1} f\right)$ for $f \in H^{2 m}(\Omega)$ with $m \geq 2$. Depending on the context, $\mathbb{1}$ denotes either the identity mapping or the identity matrix. For a matrix $A \in \mathbb{R}^{n, n}$, we refer to $A^{\text {sym }}=\frac{1}{2}\left(A+A^{T}\right)$ as the symmetric part of $A$. The symbol ":" indicates the sum over all pairwise products of two tensors. Finally, we denote the variational derivative of a functional $J$ at a point $A$ in a direction $B$ by $\partial_{A} J[A](B)=$ $\left.\frac{\mathrm{d}}{\mathrm{d} \epsilon} J[A+\epsilon B]\right|_{\epsilon=0}$.

2. Time discrete metamorphosis model. In this section, we briefly recall in a nonrigorous fashion the Riemannian geometry of the space of images based on the flow of diffeomorphisms and its extension, the metamorphosis model. For a detailed exposition of these models, we refer the reader to $[8,24,32,16,31]$. Furthermore, we introduce the time discrete metamorphosis model analyzed in [6], on which the discrete exponential map relies. In this paper, we restrict our considerations to the particular energy density function $W(D \phi)=|D \phi-\mathbb{1}|^{2}$ to circumvent lengthy term transforms and clarify the presentation. Since this energy density function does not meet the requirements of the existence theory shown in [6] and to give a self-contained presentation, an existence proof for minimizing deformations tailored to this specific energy density is shown in Proposition 2.1.

Copyright (c) by SIAM. Unauthorized reproduction of this article is prohibited. 
Flow of diffeomorphisms. Throughout this paper, we assume that the image domain $\Omega \subset \mathbb{R}^{n}$ for $n \in\{2,3\}$ has Lipschitz boundary. In the flow of diffeomorphisms approach $[2,3,8$, $4,18,22]$, one studies the temporal change of image intensities described by a family of diffeomorphisms $(\psi(t))_{t \in[0,1]}: \bar{\Omega} \rightarrow \mathbb{R}^{n}$. Here the metric and the path energy associated with the path $(\psi(t))_{t \in[0,1]}$ are given by

$$
g_{\psi(t)}(\dot{\psi}(t), \dot{\psi}(t))=\int_{\Omega} L[v(t), v(t)] \mathrm{d} x, \quad \mathcal{E}\left[(\psi(t))_{t \in[0,1]}\right]=\int_{0}^{1} g_{\psi(t)}(\dot{\psi}(t), \dot{\psi}(t)) \mathrm{d} t,
$$

where $v(t)=\dot{\psi}(t) \circ \psi^{-1}(t)$ represents the time-dependent Eulerian velocity of the underlying flow and $L$ corresponds to a quadratic form induced by a higher order elliptic operator. Let us emphasize that this path energy is right-invariant w.r.t. the group action of a diffeomorphism group, as discussed in $[8,29]$. By construction, this model comes with the brightness constancy assumption in the sense that the material derivative $\frac{D}{\partial t} u=\dot{u}+v \cdot \nabla u$ vanishes along the motion paths. From the viewpoint of physics, the metric describes the viscous dissipation in a multipolar fluid model (cf. [25, 14, 13]), in which a suitable choice for the viscous dissipation is given by a combination of a classical Newtonian flow and a simple multipolar dissipation model, namely

$$
L[v(t), v(t)]=\frac{\lambda}{2}(\operatorname{tr} \varepsilon[v])^{2}+\mu \operatorname{tr}\left(\varepsilon[v]^{2}\right)+\gamma\left|D^{m} v\right|^{2},
$$

where $\varepsilon[v]=\frac{1}{2}\left(\nabla v+\nabla v^{T}\right), m>1+\frac{n}{2}$, and $\lambda, \mu, \gamma>0$. Dupuis, Grenander, and Miller [8, Theorems 2.5 and 3.1] proved that paths of finite energy connecting the diffeomorphisms $\psi(0)=\psi_{A}$ and $\psi(1)=\psi_{B}$ are indeed one-parameter families of diffeomorphisms provided that the metric is $H^{3}(\Omega)$-coercive and an energy minimizing velocity field $v$ exists such that $\dot{\psi}(t, \cdot)=v(t, \psi(t, \cdot))$ for every $t \in[0,1]$. These diffeomorphic flows imply paths between certain pairs of image intensity functions $u_{A}, u_{B} \in L^{2}(\Omega)$ generated by the transport equation $u(t, \cdot)=u_{A} \circ \psi^{-1}(t, \cdot)$. Surely, for general pairs of input images $u_{A}, u_{B} \in L^{2}(\Omega)$ such a flow of diffeomorphism must not exist. Here the inexact LDDMM method offers a suitable generalization [18]. Since rigid body motions are generated by motion fields $v$ with spatially constant, skew-symmetric Jacobians such that $\varepsilon[v]=0$ and $D^{m} v=0$, one can easily infer the invariance of this model under rigid body motions.

Metamorphosis. The metamorphosis model [24, 32, 31] extends the flow of diffeomorphisms approach by additionally taking into account image intensity variations along motion paths. Heuristically, for a sufficiently smooth family of images $u$ the metric and the path energy in the metamorphosis model for $\delta>0$ are defined as

$$
g(\dot{u}, \dot{u})=\min _{v: \bar{\Omega} \rightarrow \mathbb{R}^{n}} \int_{\Omega} L[v, v]+\frac{1}{\delta}\left(\frac{D}{\partial t} u\right)^{2} \mathrm{~d} x, \quad \mathcal{E}[u]=\int_{0}^{1} g(\dot{u}(t), \dot{u}(t)) \mathrm{d} t .
$$

Since the same temporal change $\dot{u}(t)$ is induced by different pairs of motion fields and associated material derivatives, one has to consider equivalence classes of pairs $\left(v, \frac{D}{\partial t} u\right)$ as tangent vectors in the space of images, where such pairs are equivalent if and only if they imply the same temporal change $\dot{u}$. Hence, to evaluate the metric on such tangent vectors one has to 
minimize over the elements of the equivalence class, and computing a geodesic path requires optimizing both the temporal change of the image intensity and the motion field. In this case, the first term $L[v, v]$ reflects the cost of the underlying transport and the term $\frac{1}{\delta}\left(\frac{D}{\partial t} u\right)^{2}$ penalizes the variation of the image intensity along motion paths. Since neither images nor paths in the image space are expected to be smooth, the material derivative is not welldefined. To circumvent this issue, we use a nonlinear geometric structure in the space of images $L^{2}(\Omega):=L^{2}(\Omega, \mathbb{R})$ following Trouvé and Younes [31], in which the material derivative is replaced by a weak version. In detail, for $v \in L^{2}\left((0,1), H^{m}\left(\Omega, \mathbb{R}^{n}\right) \cap H_{0}^{1}\left(\Omega, \mathbb{R}^{n}\right)\right)$ the function $z \in L^{2}\left((0,1), L^{2}(\Omega)\right)$ is defined as the weak material derivative of an image path $u \in L^{2}\left((0,1), L^{2}(\Omega)\right)$ if

$$
\int_{0}^{1} \int_{\Omega} \eta z \mathrm{~d} x \mathrm{~d} t=-\int_{0}^{1} \int_{\Omega}\left(\partial_{t} \eta+\operatorname{div}(v \eta)\right) u \mathrm{~d} x \mathrm{~d} t
$$

for $\eta \in C_{c}^{\infty}((0,1) \times \Omega)$. Then the tangent space $T_{u} L^{2}(\Omega)$ at $u \in L^{2}(\Omega)$ is given by $T_{u} L^{2}(\Omega)=$ $\{u\} \times W / N_{u}$, where $W=\left(H^{m}\left(\Omega, \mathbb{R}^{n}\right) \cap H_{0}^{1}\left(\Omega, \mathbb{R}^{n}\right)\right) \times L^{2}(\Omega)$ and

$$
N_{u}=\left\{w=(v, z) \in W: \int_{\Omega} z \eta+u \operatorname{div}(\eta v) \mathrm{d} x=0 \forall \eta \in C_{c}^{\infty}(\Omega)\right\}
$$

and we denote equivalence classes in this space by $(u, \overline{(v, z)})$. Hence, the associated tangent bundle is $T L^{2}(\Omega)=\bigcup_{u \in L^{2}(\Omega)} T_{u} L^{2}(\Omega)$.

Incorporating this geometric structure, one can define a regular path in the space of images in a weak sense as follows: a curve $u \in C^{0}\left([0,1], L^{2}(\Omega)\right)$ is regular in the space of images (denoted by $u \in H^{1}\left((0,1), L^{2}(\Omega)\right)$ ) if there exists a measurable path $\gamma:[0,1] \rightarrow T L^{2}(\Omega)$ with bounded $L^{2}$-norm in space and time and $\pi(\gamma)=u$, where $\pi(u, \overline{(v, z)})=u$ is the projection onto the image manifold, such that

$$
-\int_{0}^{1} \int_{\Omega} u \partial_{t} \eta \mathrm{d} x \mathrm{~d} t=\int_{0}^{1} \int_{\Omega} z \eta+u \operatorname{div}(\eta v) \mathrm{d} x \mathrm{~d} t
$$

for all $\eta \in C_{c}^{\infty}((0,1) \times \Omega)$. Then the path energy in the metamorphosis model for a regular path $u \in H^{1}\left((0,1), L^{2}(\Omega)\right)$ and for the quadratic form $L$ being coercive on $H^{m}\left(\Omega, \mathbb{R}^{n}\right) \cap H_{0}^{1}\left(\Omega, \mathbb{R}^{n}\right)$ reads as

$$
\mathcal{E}[u]=\int_{0}^{1} \inf _{(v, z) \in T_{u(t)} L^{2}(\Omega)} \int_{\Omega} L[v, v]+\frac{1}{\delta} z^{2} \mathrm{~d} x \mathrm{~d} t .
$$

In [31, Theorems 2 and 6], Trouvé and Younes proved the existence of minimizing paths for given boundary data in time; i.e., for given images $u_{A}, u_{B} \in L^{2}(\Omega)$, there exists a curve $u \in H^{1}\left((0,1), L^{2}(\Omega)\right)$ with $u(0)=u_{A}$ and $u(1)=u_{B}$ such that

$$
\mathcal{E}[u]=\inf \left\{\mathcal{E}[\tilde{u}]: \tilde{u} \in H^{1}\left((0,1), L^{2}(\Omega)\right), \tilde{u}(0)=u_{A}, \tilde{u}(1)=u_{B}\right\}
$$

and there exist minimizing $(v, z) \in T_{u(t)} L^{2}(\Omega)$ provided that $m>1+\frac{n}{2}$ and $\gamma, \delta>0$. Finally, let us remark that the flow of diffeomorphisms model can be considered as the limit case of the metamorphosis model when letting $\delta \searrow 0$.

Copyright $\odot$ by SIAM. Unauthorized reproduction of this article is prohibited. 
Time discrete metamorphosis model. In what follows, we will briefly recall the time discretization approach for the time continuous path energy (2.4) originally developed in [6] and based on the general theory of a discrete geodesic calculus on shape spaces developed in [28]. The building block is a functional $\mathcal{W}$, which is considered as an easy to evaluate approximation of the squared Riemannian distance on the shape space under consideration. In the case of the metamorphosis model, we define for $\gamma, \delta>0$ and $m>1+\frac{n}{2}$ the functional

$$
\mathcal{W}[u, \tilde{u}]=\min _{\phi \in \mathcal{A}} \int_{\Omega} W(D \phi)+\gamma\left|D^{m} \phi\right|^{2}+\frac{1}{\delta}|\tilde{u} \circ \phi-u|^{2} \mathrm{~d} x,
$$

where $\mathcal{A}=\left\{\phi \in H^{m}(\Omega, \Omega): \operatorname{det} D \phi>0\right.$ a.e. in $\Omega, \phi=\mathbb{1}$ on $\left.\partial \Omega\right\}$ is the set of admissible deformations, $u, \tilde{u} \in L^{2}(\Omega)$ are arbitrary images, and $W: \mathbb{R}^{n, n} \rightarrow \mathbb{R}$ is an energy density function, which is assumed to be nonnegative and polyconvex and satisfies $W(A) \geq \beta_{0}(\operatorname{det} A)^{-s}-\beta_{1}$ for $\beta_{0}, \beta_{1}, s>0$ and all $A \in \mathbb{R}^{n, n}$ with $\operatorname{det}(A)>0, W(A)=\infty$ for $\operatorname{det}(A) \leq 0$. Following [6, Proposition 3.1], these assumptions are already sufficient to guarantee the existence of a minimizing deformation in (2.5) and thus the well-posedness of the discrete energy $\mathcal{W}$.

Following the general approach for the variational time discretization of geodesic calculus in [28] and the particular discretization of the metamorphosis model in [6], we define the discrete path energy $\mathbf{E}_{K}$ on a sequence of $K+1$ images $\left(u_{0}, \ldots, u_{K}\right) \in\left(L^{2}(\Omega)\right)^{K+1}$ with $K \geq 2$ as the weighted sum of the discrete matching energy evaluated at consecutive images, i.e.,

$$
\mathbf{E}_{K}\left[u_{0}, \ldots, u_{K}\right]:=K \sum_{k=1}^{K} \mathcal{W}\left[u_{k-1}, u_{k}\right]
$$

A $(K+1)$-tuple $\left(u_{0}, \ldots, u_{K}\right) \in\left(L^{2}(\Omega)\right)^{K+1}$ with given images $u_{0}$ and $u_{K}$ is defined to be a discrete geodesic curve connecting $u_{0}$ and $u_{K}$ if it minimizes $\mathbf{E}_{K}$ w.r.t. all other $(K+1)$-tuples with $u_{0}$ and $u_{K}$ fixed. For the existence proof for discrete geodesics we refer the reader to [6, Theorem 3.4]. It is also shown in [6] that a suitable extension of the discrete path energy $\mathbf{E}_{K}$ $\Gamma$-converges to the continuous path energy $\mathcal{E}$ provided that the energy density $W$ and the differential operator $L$ are consistent, i.e., $W(\mathbb{1})=0, D W(\mathbb{1})=0$, and $\frac{1}{2} D^{2} W(\mathbb{1})(B, B)=$ $\frac{\lambda}{2}(\operatorname{tr} B)^{2}+\mu \operatorname{tr}\left(\left(\frac{B+B^{T}}{2}\right)^{2}\right)$ for all $B \in \mathbb{R}^{n, n}$. Let us emphasize that neither the optimal matching deformation in (2.5) nor the discrete geodesic curve for given input images $u_{0}$ and $u_{K}$ is necessarily unique.

Modified energy functional in the time discrete metamorphosis model. In this paragraph, we introduce a slightly modified version of the time discrete metamorphosis model used throughout this paper and prove its local well-posedness. By using this modification, we avoid lengthy technical term transforms and we remark that all results regarding the local existence and local uniqueness of the discrete exponential map (see section 4) remain valid in the more general case with minor modifications. The model modifications and the associated modifications of the function spaces are as follows:

- We consider the particular differential operator

$$
L[v, v]=D v: D v+\gamma \Delta^{m} v \cdot \Delta^{m} v
$$


with $\gamma>0$ and $2 m>1+\frac{n}{2}$, which is linked to the energy density $W(D \phi)=|D \phi-\mathbb{1}|^{2}$ and the higher order regularization term $\gamma\left|\Delta^{m} \phi\right|^{2}$.

- The set of admissible deformations $\mathcal{A}$ is defined as

$$
\mathcal{A}=\left\{\phi \in H^{2 m}(\Omega, \Omega): \phi-\mathbb{1} \in H_{0}^{2 m}(\Omega, \Omega)\right\} .
$$

In the model presented in the preceding paragraph, the weaker boundary condition $\phi=\mathbb{1}$ on $\partial \Omega$ instead of $\phi-\mathbb{1} \in H_{0}^{2 m}(\Omega, \Omega)$ was assumed for all deformations $\phi$. Here this stronger condition is required for both a higher regularity result (cf. Proposition 3.2) and a higher order control of the deformations (cf. (4.1)). With these altered boundary conditions the equality

$$
\int_{\Omega}\left|\Delta^{m} \psi\right|^{2} \mathrm{~d} x=\int_{\Omega}\left|D^{2 m} \psi\right|^{2} \mathrm{~d} x \quad \text { for all } \psi \in H_{0}^{2 m}(\Omega, \Omega)
$$

holds true for all $m \geq 1$ (cf. [11, section 2.2]). In fact, using integration by parts we exemplarily obtain for $m=1$

$$
\int_{\Omega}|\Delta \psi|^{2} \mathrm{~d} x=\int_{\Omega} \sum_{i, j=1}^{n} \partial_{i}^{2} \psi \cdot \partial_{j}^{2} \psi \mathrm{d} x=\int_{\Omega} \sum_{i, j=1}^{n} \partial_{i} \partial_{j} \psi \cdot \partial_{i} \partial_{j} \psi \mathrm{d} x=\int_{\Omega}\left|D^{2} \psi\right|^{2} \mathrm{~d} x .
$$

To sum up, for arbitrary images $u, \tilde{u} \in L^{2}(\Omega)$ the discrete pairwise matching energy is defined as

$$
\mathcal{W}[u, \tilde{u}]=\min _{\phi \in \mathcal{A}}\left\{\mathcal{W}^{D}[u, \tilde{u}, \phi]:=\int_{\Omega}|D \phi-\mathbb{1}|^{2}+\gamma\left|\Delta^{m} \phi\right|^{2}+\frac{1}{\delta}(\tilde{u} \circ \phi-u)^{2} \mathrm{~d} x\right\},
$$

which is composed of a rescaled thin plate regularization term (first two terms) and a quadratic $L^{2}(\Omega)$-mismatch measure (cf. [6, equation (6.2)]).

In what follows, we will prove the existence of minimizers of this particular matching energy $\mathcal{W}$ for input images $u, \tilde{u} \in L^{2}(\Omega)$ with $\|\tilde{u}-u\|_{L^{2}(\Omega)}$ being sufficiently small.

Proposition 2.1 (existence of a minimizing deformation for $\mathcal{W}$ ). Let $u \in L^{2}(\Omega)$ and $2 m-\frac{n}{2}>$ 1. Then there exists a constant $C_{\mathcal{W}}>0$ that solely depends on $\gamma, \delta, \Omega$, and $m$ such that for every $\tilde{u} \in L^{2}(\Omega)$ with $\|\tilde{u}-u\|_{L^{2}(\Omega)} \leq C_{\mathcal{W}}$ there is a minimizing deformation $\phi \in \mathcal{A}$ for $\mathcal{W}$, i.e., $\mathcal{W}[u, \tilde{u}]=\mathcal{W}^{D}[u, \tilde{u}, \phi]$, and $\phi$ is a $C^{1}(\Omega)$-diffeomorphism.

Proof. The proof is based on the direct method in the calculus of variations. Let $\left(\phi^{j}\right)_{j \in \mathbb{N}} \subset$ $\mathcal{A}$ be a minimizing sequence for $\mathcal{W}^{D}\left[u, \tilde{u}, \phi^{j}\right]$ with monotonously decreasing energy, and let

$$
0 \leq \inf _{\tilde{\phi} \in \mathcal{A}} \mathcal{W}^{D}[u, \tilde{u}, \tilde{\phi}]=\lim _{j \rightarrow \infty} \mathcal{W}^{D}\left[u, \tilde{u}, \phi^{j}\right] \leq \overline{\mathbf{W}}:=\mathcal{W}^{D}[u, \tilde{u}, \mathbb{1}]=\frac{1}{\delta}\|\tilde{u}-u\|_{L^{2}(\Omega)}^{2}
$$

Since $\phi^{j}-\mathbb{1} \in H_{0}^{2 m}(\Omega),(2.7)$ and (2.9) imply

$$
\int_{\Omega} \gamma\left|D^{2 m}\left(\phi^{j}-\mathbb{1}\right)\right|^{2} \mathrm{~d} x=\int_{\Omega} \gamma\left|\Delta^{m}\left(\phi^{j}-\mathbb{1}\right)\right|^{2} \mathrm{~d} x=\int_{\Omega} \gamma\left|\Delta^{m} \phi^{j}\right|^{2} \mathrm{~d} x \leq \frac{1}{\delta}\|\tilde{u}-u\|_{L^{2}(\Omega)}^{2} \leq \frac{C_{\mathcal{W}}^{2}}{\delta} .
$$

Thus, the norm equivalence of $\|\cdot\|_{H^{2 m}(\Omega)}$ and $|\cdot|_{H^{2 m}(\Omega)}$ for the space $H_{0}^{2 m}(\Omega)$, which follows by an iterative application of the Poincaré inequality (cf. [1, Corollary 6.31]), yields

$$
\left\|\phi^{j}-\mathbb{1}\right\|_{H^{2 m}(\Omega)} \leq C\|\tilde{u}-u\|_{L^{2}(\Omega)} \leq C C_{\mathcal{W}} .
$$

Copyright (C) by SIAM. Unauthorized reproduction of this article is prohibited. 
By taking into account the embedding $H^{2 m}(\Omega) \hookrightarrow C^{1}(\bar{\Omega})$ and considering a smaller $C_{\mathcal{W}}$ if necessary, we can assume

$$
\left\|\operatorname{det}\left(D \phi^{j}\right)-1\right\|_{L^{\infty}(\Omega)} \leq C_{d}
$$

for a constant $C_{d} \in(0,1)$, which implies that $\phi^{j}$ is a $C^{1}(\Omega)$-diffeomorphism (see [7, Theorem 5.5-2]). Moreover, since $\left(\phi^{j}\right)_{j \in \mathbb{N}}$ are uniformly bounded in $H^{2 m}(\Omega)$ (cf. (2.10)), a subsequence (also denoted by $\phi^{j}$ ) converges weakly in $H^{2 m}(\Omega)$ due to the reflexivity of this space and (strongly) in $C^{1, \alpha}(\bar{\Omega})$ for $\alpha \in\left(0,2 m-1-\frac{n}{2}\right)$ to a $C^{1}(\Omega)$-diffeomorphism $\phi \in \mathcal{A}$.

Next, we prove the convergence of the $L^{2}(\Omega)$-mismatch terms. To this end, we estimate

$$
\begin{aligned}
& \left|\int_{\Omega}\right| \tilde{u} \circ \phi^{j}-\left.u\right|^{2}-|\tilde{u} \circ \phi-u|^{2} \mathrm{~d} x\left|\leq \int_{\Omega}\left(\left|\tilde{u} \circ \phi^{j}-u\right|+|\tilde{u} \circ \phi-u|\right)\right| \tilde{u} \circ \phi^{j}-\tilde{u} \circ \phi \mid \mathrm{d} x \\
\leq & \left(\left\|\tilde{u} \circ \phi^{j}-u\right\|_{L^{2}(\Omega)}+\|\tilde{u} \circ \phi-u\|_{L^{2}(\Omega)}\right)\left\|\tilde{u} \circ \phi^{j}-\tilde{u} \circ \phi\right\|_{L^{2}(\Omega)} \\
\leq & 2 \sqrt{\delta \overline{\mathbf{W}}}\left\|\tilde{u} \circ \phi^{j}-\tilde{u} \circ \phi\right\|_{L^{2}(\Omega)} .
\end{aligned}
$$

In the next step, we approximate $\tilde{u}$ in $L^{2}(\Omega)$ by a sequence of smooth functions $\left(\tilde{u}_{i}\right)_{i \in \mathbb{N}}$ such that $\left\|\tilde{u}-\tilde{u}_{i}\right\|_{L^{2}(\Omega)} \leq 2^{-i}$. Then

$$
\left\|\tilde{u} \circ \phi^{j}-\tilde{u} \circ \phi\right\|_{L^{2}(\Omega)} \leq\left\|\tilde{u} \circ \phi^{j}-\tilde{u}_{i} \circ \phi^{j}\right\|_{L^{2}(\Omega)}+\left\|\tilde{u}_{i} \circ \phi^{j}-\tilde{u}_{i} \circ \phi\right\|_{L^{2}(\Omega)}+\left\|\tilde{u}_{i} \circ \phi-\tilde{u} \circ \phi\right\|_{L^{2}(\Omega)} .
$$

Next, applying the transformation formula yields

$$
\left\|\tilde{u} \circ \phi^{j}-\tilde{u}_{i} \circ \phi^{j}\right\|_{L^{2}(\Omega)} \leq\left\|\left(\operatorname{det}\left(D \phi^{j}\right) \circ\left(\phi^{j}\right)^{-1}\right)^{-1}\right\|_{L^{\infty}(\Omega)}^{\frac{1}{2}}\left\|\tilde{u}-\tilde{u}_{i}\right\|_{L^{2}(\Omega)} \leq \frac{1}{\left(1-C_{d}\right)^{\frac{1}{2}}}\left\|\tilde{u}-\tilde{u}_{i}\right\|_{L^{2}(\Omega)} .
$$

Likewise, we can deduce $\left\|\tilde{u} \circ \phi-\tilde{u}_{i} \circ \phi\right\|_{L^{2}(\Omega)} \leq C\left\|\tilde{u}-\tilde{u}_{i}\right\|_{L^{2}(\Omega)}$. Furthermore, the middle term in (2.11) vanishes for fixed $i$ as $j \rightarrow \infty$. Finally, using the lower semicontinuity of the first two terms of the energy we get

$$
\mathcal{W}^{D}[u, \tilde{u}, \phi] \leq \liminf _{j \rightarrow \infty} \mathcal{W}^{D}\left[u, \tilde{u}, \phi^{j}\right],
$$

which proves this proposition.

3. The time discrete exponential map. In this section, we define the discrete exponential map and derive optimality as well as regularity results, on which the study of the existence and the uniqueness in section 4 and the algorithm introduced in section 5 will be based.

Let us briefly recall the definition of the continuous exponential map on a Riemannian manifold. Let $y:[0,1] \rightarrow \mathcal{M}$ be the unique geodesic curve for a prescribed initial position $y(0)=y_{A}$ and an initial velocity $\dot{y}(0)=v$ on a Riemannian manifold $(\mathcal{M}, g)$. The exponential map is then defined $\operatorname{as}_{\exp _{y_{A}}}(v)=y(1)$. Furthermore, one easily checks that $\exp _{y_{A}}\left(\frac{k}{K} v\right)=$ $y\left(\frac{k}{K}\right)$ for $0 \leq k \leq K$. We refer the reader to the textbook [19] for a detailed discussion of the (continuous) exponential map. Now we ask for a time discrete counterpart of the exponential map in the metamorphosis model. To this end, we consider an image $u_{0}$ as the initial data and a second image $u_{1}$ such that $\zeta_{1}=u_{1}-u_{0}$ represents a small variation of the image $u_{0}$. This variation $\zeta_{1}$ is the discrete counterpart of the infinitesimal variation given by the velocity 
$v$ in the continuous case. For varying values of $K \geq 2$ we now ask for a discrete geodesic $\left(u_{0}, u_{1}, u_{2}, \ldots, u_{K}\right)$ described as the minimizer of the discrete path energy (2.6). Let us for the time being suppose that this geodesic is unique - a property to be verified later. Based on our above observation for the continuous exponential map, we define $\operatorname{EXP}_{*}^{k}(\cdot)$ as the discrete counterpart of $\exp _{*}\left(\frac{k}{K} \cdot\right)$; i.e., we set

$$
\operatorname{EXP}_{u_{0}}^{k}\left(\zeta_{1}\right):=u_{k}
$$

for $k=1, \ldots, K$. The definition of the exponential map $\operatorname{EXP}_{u_{0}}^{k}\left(\zeta_{1}\right)$ does not depend on the number of time steps $K$. Indeed, if $\left(u_{0}, u_{1}, u_{2}, \ldots, u_{K}\right)$ is a discrete geodesic, then $\left(u_{0}, u_{1}, u_{2}, \ldots, u_{L}\right)$ with $L \leq K$ is also a geodesic. Taking into account $k=2$ we immediately observe that the sequence of discrete exponential maps $\left(\operatorname{EXP}_{u_{0}}^{k}\left(\zeta_{1}\right)\right)_{k=1, \ldots}$ can iteratively be defined as follows:

$$
\operatorname{EXP}_{u_{0}}^{k}\left(\zeta_{1}\right)=u_{k}:=\operatorname{EXP}_{u_{k-2}}^{2}\left(\zeta_{k-1}\right)
$$

for $k \geq 2$, where $\zeta_{k-1}=u_{k-1}-u_{k-2}$, and for the sake of completeness we define $\operatorname{EXP}_{u_{0}}^{0}\left(\zeta_{1}\right)=$ $u_{0}$ and $\operatorname{EXP}_{u_{0}}^{1}\left(\zeta_{1}\right)=u_{1}=u_{0}+\zeta_{1}$. Thus, it essentially remains to compute $\operatorname{EXP}^{2}$ for a given input image $u_{k-2}$ and an image variation $\zeta_{k-1}=u_{k-1}-u_{k-2}$ (see Figure 1). For a detailed discussion of the discrete exponential map in the simpler model of Hilbert manifolds we refer the reader to [28]. The particular challenge here is that the matching energy $\mathcal{W}$ cannot be evaluated directly but requires solving the variational problem (2.8) for the matching deformation.

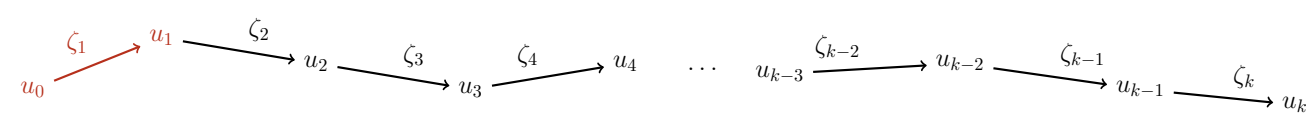

Figure 1. Schematic drawing of $\operatorname{EXP}_{u_{0}}^{k}\left(\zeta_{1}\right), k=1, \ldots, K$; the input data is highlighted in red.

There are two major restrictions regarding the input images $u_{0}$ and $u_{1}$.

First, the existence and uniqueness result for the discrete exponential map (cf. section 4) will require weakly differentiable input images. These weak derivatives of images naturally arise in the Euler-Lagrange equations for $\mathrm{EXP}^{2}$ w.r.t. the deformations (see (3.5) and (3.6) below). Let us remark that weak differentiability of the input data for the exponential map is also a crucial requirement in the initial value problem for the geodesic equation in [31]. Furthermore, the $H^{1}(\Omega)$-regularity property is inherited along discrete geodesics; i.e., $\operatorname{EXP}_{u_{0}}^{k}\left(u_{1}-u_{0}\right) \in H^{1}(\Omega)$ for any $k \geq 1$ provided that $u_{0}, u_{1} \in H^{1}(\Omega)$ (cf. [6, Remark 3.3 and equation (3.2)]).

Second, the initial variation $\zeta_{1}=u_{1}-u_{0}$ is assumed to be sufficiently small in $L^{2}(\Omega)$ in order to ensure the existence of the initial deformation $\phi_{1}$ and guarantee the convergence of a suitable fixed point algorithm - a property which appears to be natural in light of the analog assumption for the continuous exponential map [19]. We will also see that for fixed $K$ the variations $u_{k-1}-u_{k-2}$ for $k \leq K$ will remain small provided that $\zeta_{1}$ is small. Thus, for fixed $K$ the discrete exponential map $\operatorname{EXP}_{u_{0}}^{k}(\cdot)$ will be well-posed for a sufficiently small initial variation $\zeta_{1}$. 
Hence, in what follows we consider images in $H^{1}(\Omega)$ and define $u_{k}:=\operatorname{EXP}_{u_{k-2}}^{2}\left(\zeta_{k-1}\right)$ as the (unique) image in $H^{1}(\Omega)$ such that

$$
u_{k-1}=\underset{u \in H^{1}(\Omega)}{\operatorname{argmin}} \min _{\phi_{k-1}, \phi_{k} \in \mathcal{A}} \mathcal{W}^{D}\left[u_{k-2}, u, \phi_{k-1}\right]+\mathcal{W}^{D}\left[u, u_{k}, \phi_{k}\right]
$$

For the sake of simplicity, we restrict ourselves to the first step in the iterative computation of the discrete exponential map with $k=2$. Given $u_{0}, u_{1} \in H^{1}(\Omega)$, the first order optimality conditions for (3.2) for $u_{2} \in H^{1}(\Omega)$ and $\phi_{1}, \phi_{2} \in \mathcal{A}$ read as

$$
\begin{aligned}
\partial_{u_{1}}\left(\mathcal{W}^{D}\left[u_{0}, u_{1}, \phi_{1}\right]+\mathcal{W}^{D}\left[u_{1}, u_{2}, \phi_{2}\right]\right)(v) & =0, \\
\partial_{\phi_{1}} \mathcal{W}^{D}\left[u_{0}, u_{1}, \phi_{1}\right](\psi) & =0, \\
\partial_{\phi_{2}} \mathcal{W}^{D}\left[u_{1}, u_{2}, \phi_{2}\right](\psi) & =0
\end{aligned}
$$

for all $v \in H^{1}(\Omega)$ and all $\psi \in H_{0}^{2 m}(\Omega)$ for $2 m-\frac{n}{2}>2$. The system (3.3) is equivalent to

$$
\begin{array}{r}
\int_{\Omega}\left(u_{1} \circ \phi_{1}-u_{0}\right) v \circ \phi_{1}-\left(u_{2} \circ \phi_{2}-u_{1}\right) v \mathrm{~d} x=0, \\
\int_{\Omega} 2 D \phi_{1}: D \psi+2 \gamma \Delta^{m} \phi_{1} \cdot \Delta^{m} \psi+\frac{2}{\delta}\left(u_{1} \circ \phi_{1}-u_{0}\right)\left(\nabla u_{1} \circ \phi_{1}\right) \cdot \psi \mathrm{d} x=0, \\
\int_{\Omega} 2 D \phi_{2}: D \psi+2 \gamma \Delta^{m} \phi_{2} \cdot \Delta^{m} \psi+\frac{2}{\delta}\left(u_{2} \circ \phi_{2}-u_{1}\right)\left(\nabla u_{2} \circ \phi_{2}\right) \cdot \psi \mathrm{d} x=0 .
\end{array}
$$

The subsequent lemma provides a reformulation of the above system of equations, in which the dependency of the unknown function $u_{2}$ in (3.6) is removed and in addition solely the function $u_{1}$ and no longer derivatives of $u_{1}$ appear.

Lemma 3.1 (reformulation of the Euler-Lagrange equation for $\phi_{2}$ ). Let $u_{0}, u_{1}, u_{2} \in H^{1}(\Omega)$ such that (cf. Proposition 2.1)

$$
\left\|u_{1}-u_{0}\right\|_{L^{2}(\Omega)},\left\|u_{2}-u_{1}\right\|_{L^{2}(\Omega)} \leq C_{\mathcal{W}}
$$

$2 m-\frac{n}{2}>2$, and assume that (3.4) and (3.5) hold true. Let $\phi_{i}$ with $i=1,2$ be the minimizer of $\mathcal{W}^{D}\left[u_{i-1}, u_{i}, \cdot\right]$ on $\mathcal{A}$ according to Proposition 2.1:

(i) Then (3.6) is equivalent to

$$
\begin{aligned}
& \int_{\Omega} 2 \gamma \Delta^{m} \phi_{2} \cdot \Delta^{m} \psi+2 D \phi_{2}: D \psi+\frac{2}{\delta}\left(u_{1} \circ \phi_{1}-u_{0}\right)\left(\nabla u_{1} \cdot\left(D \phi_{2}\right)^{-1} \psi\right) \circ \phi_{1} \\
& +\frac{1}{\delta} \frac{\left(u_{1} \circ \phi_{1}-u_{0}\right)^{2}}{\operatorname{det} D \phi_{1}}\left(\left(D \phi_{2}\right)^{-T}:\left(D^{2} \phi_{2}\left(D \phi_{2}\right)^{-1} \psi\right)-\left(D \phi_{2}\right)^{-T}: D \psi\right) \circ \phi_{1} \mathrm{~d} x=0
\end{aligned}
$$

for all $\psi \in H_{0}^{2 m}(\Omega)$. 
(ii) Under the additional assumptions that $\partial \Omega \in C^{4 m}$ and $u_{0}, u_{1}, u_{2} \in L^{\infty}(\Omega) \cap H^{1}(\Omega)$, (3.6) is equivalent to

$$
\begin{aligned}
& \int_{\Omega} 2 \gamma \Delta^{m} \phi_{2} \cdot \Delta^{m} \psi+2 D \phi_{2}: D \psi \mathrm{d} x \\
= & \int_{\Omega} 2 \gamma \Delta^{m} \phi_{1} \cdot \Delta^{m}\left(\left(\left(D \phi_{2}\right)^{-1} \psi\right) \circ \phi_{1}\right)+2 D \phi_{1}: D\left(\left(\left(D \phi_{2}\right)^{-1} \psi\right) \circ \phi_{1}\right) \\
& -\frac{1}{\delta} \frac{\left(u_{1} \circ \phi_{1}-u_{0}\right)^{2}}{\operatorname{det} D \phi_{1}}\left(\left(D \phi_{2}\right)^{-T}:\left(D^{2} \phi_{2}\left(D \phi_{2}\right)^{-1} \psi\right)-\left(D \phi_{2}\right)^{-T}: D \psi\right) \circ \phi_{1} \mathrm{~d} x .
\end{aligned}
$$

Here the notation $\left(D^{2} \phi_{2}\left(D \phi_{2}\right)^{-1} \psi\right)_{j k}=\sum_{i, l=1}^{n} \partial_{j} \partial_{k} \phi_{2}^{i}\left(D \phi_{2}\right)_{i l}^{-1} \psi_{l}$ is used.

Proof. By using the transformation formula the energy $\mathcal{W}^{D}$ can be rewritten as follows:

$$
\mathcal{W}^{D}\left[u_{1}, u_{2}, \phi_{2}\right]=\int_{\Omega}\left|D \phi_{2}-\mathbb{1}\right|^{2}+\gamma\left|\Delta^{m} \phi_{2}\right|^{2}+\frac{1}{\delta} \frac{\left(u_{2}-u_{1} \circ \phi_{2}^{-1}\right)^{2}}{\operatorname{det}\left(D \phi_{2}\right) \circ \phi_{2}^{-1}} \mathrm{~d} x
$$

since $\phi_{2} \in \mathcal{A}$ is a diffeomorphism (see Proposition 2.1). As the next step, we rewrite the EulerLagrange equation w.r.t. $\phi_{2}$ of $\mathcal{W}^{D}\left[u_{1}, u_{2}, \cdot\right]$. To this end, we use the identities $\partial_{\phi_{2}} \phi_{2}^{-1}(\psi)=$ $-\left(\left(D \phi_{2}\right)^{-1} \psi\right) \circ \phi_{2}^{-1}$, which follows by differentiating $\left(\phi_{2}+\epsilon \psi\right) \circ\left(\phi_{2}+\epsilon \psi\right)^{-1}=\mathbb{1}$ w.r.t. $\epsilon$, and $\partial_{A} \operatorname{det}(A)(B)=\operatorname{cof}(A): B$ for $A \in G L(n)$ and $B \in \mathbb{R}^{n, n}$ with $\operatorname{cof} A=(\operatorname{det} A) A^{-T}$. Thus, we obtain

$$
\begin{aligned}
& \int_{\Omega} 2 D \phi_{2}: D \psi+2 \gamma \Delta^{m} \phi_{2} \cdot \Delta^{m} \psi+\frac{2}{\delta}\left(u_{2}-u_{1} \circ \phi_{2}^{-1}\right) \frac{\left(\nabla u_{1} \cdot\left(D \phi_{2}\right)^{-1} \psi\right) \circ \phi_{2}^{-1}}{\operatorname{det}\left(D \phi_{2}\right) \circ \phi_{2}^{-1}} \\
& +\frac{1}{\delta} \frac{\left(u_{2}-u_{1} \circ \phi_{2}^{-1}\right)^{2}}{\left(\operatorname{det} D \phi_{2}\right)^{2} \circ \phi_{2}^{-1}}\left(\operatorname{cof} D \phi_{2}:\left(D^{2} \phi_{2}\left(D \phi_{2}\right)^{-1} \psi\right)-\operatorname{cof} D \phi_{2}: D \psi\right) \circ \phi_{2}^{-1} \mathrm{~d} x=0 .
\end{aligned}
$$

A further application of the transformation formula w.r.t. $\phi_{2}$ yields

$$
\begin{gathered}
\int_{\Omega} 2 D \phi_{2}: D \psi+2 \gamma \Delta^{m} \phi_{2} \cdot \Delta^{m} \psi+\frac{2}{\delta}\left(u_{2} \circ \phi_{2}-u_{1}\right) \nabla u_{1} \cdot\left(D \phi_{2}\right)^{-1} \psi \\
+\frac{1}{\delta} \frac{\left(u_{2} \circ \phi_{2}-u_{1}\right)^{2}}{\operatorname{det} D \phi_{2}}\left(\operatorname{cof} D \phi_{2}:\left(D^{2} \phi_{2}\left(D \phi_{2}\right)^{-1} \psi\right)-\operatorname{cof} D \phi_{2}: D \psi\right) \mathrm{d} x=0 .
\end{gathered}
$$

To remove the dependency of the function $u_{2}$ above, we employ the pointwise condition

$$
u_{2} \circ \phi_{2}-u_{1}=\frac{u_{1}-u_{0} \circ \phi_{1}^{-1}}{\operatorname{det}\left(D \phi_{1}\right) \circ \phi_{1}^{-1}}
$$

for a.e. $x \in \Omega$, which follows directly from (3.4). Inserting this in (3.9) and using the integral transformation formula we achieve

$$
\int_{\Omega} 2 D \phi_{2}: D \psi+2 \gamma \Delta^{m} \phi_{2} \cdot \Delta^{m} \psi+\frac{2}{\delta}\left(u_{1} \circ \phi_{1}-u_{0}\right)\left(\nabla u_{1} \cdot\left(D \phi_{2}\right)^{-1} \psi\right) \circ \phi_{1}
$$

Copyright (C) by SIAM. Unauthorized reproduction of this article is prohibited. 


$$
+\frac{1}{\delta} \frac{\left(u_{1} \circ \phi_{1}-u_{0}\right)^{2}}{\operatorname{det} D \phi_{1}}\left(\frac{\operatorname{cof} D \phi_{2}:\left(D^{2} \phi_{2}\left(D \phi_{2}\right)^{-1} \psi\right)-\operatorname{cof} D \phi_{2}: D \psi}{\operatorname{det} D \phi_{2}}\right) \circ \phi_{1} \mathrm{~d} x=0 .
$$

The identity $\operatorname{cof}(A)=\operatorname{det}(A) A^{-T}$ for $A \in G L(n)$ implies (i).

To show (ii), we take into account the test function $\zeta:=\left(\left(D \phi_{2}\right)^{-1} \psi\right) \circ \phi_{1}$ in (3.5). To justify this, we have to show that $\zeta \in H_{0}^{2 m}(\Omega)$. To this end, we require $H^{2 m+1}(\Omega)$-regularity of $\phi_{2}$, which will follow from Proposition 3.2, and classical differential calculus for Sobolev functions [1]. Inserting $\zeta$ into (3.5) we get

$$
\begin{aligned}
& -\int_{\Omega} \frac{2}{\delta}\left(u_{1} \circ \phi_{1}-u_{0}\right)\left(\nabla u_{1} \cdot\left(D \phi_{2}\right)^{-1} \psi\right) \circ \phi_{1} \mathrm{~d} x \\
= & \int_{\Omega} 2 \gamma \Delta^{m} \phi_{1} \cdot \Delta^{m}\left(\left(\left(D \phi_{2}\right)^{-1} \psi\right) \circ \phi_{1}\right)+2 D \phi_{1}: D\left(\left(\left(D \phi_{2}\right)^{-1} \psi\right) \circ \phi_{1}\right) \mathrm{d} x .
\end{aligned}
$$

By adding the above equation to (3.7) we have proven (ii).

Proposition 3.2 (maximal regularity of the deformations). Let $2 m-\frac{n}{2}>2$ and $\partial \Omega \in C^{4 m}$. Furthermore, let $u_{0}, u_{1}, u_{2} \in L^{\infty}(\Omega) \cap H^{1}(\Omega)$, and suppose that $\phi_{1}, \phi_{2} \in \mathcal{A}$ are minimizers of $\mathcal{W}^{D}\left[u_{0}, u_{1}, \cdot\right]$ and $\mathcal{W}^{D}\left[u_{1}, u_{2}, \cdot\right]$, respectively. Then $\phi_{1}, \phi_{2} \in \mathcal{A} \cap H^{4 m}(\Omega)$.

Proof. We only prove the result for $\phi_{2}$; for $\phi_{1}$, one proceeds analogously. Let $w$ be the displacement associated with $\phi_{2}$, i.e., $w=\phi_{2}-\mathbb{1} \in H_{0}^{2 m}(\Omega)$. Using integration by parts in (3.6) we obtain for a test function $\psi \in H_{0}^{2 m}(\Omega)$

$$
\begin{aligned}
& \int_{\Omega} \Delta^{m} w \cdot \Delta^{m} \psi \mathrm{d} x=-\int_{\Omega} \frac{1}{\gamma \delta}\left(u_{2} \circ \phi_{2}-u_{1}\right)\left(\left(\nabla u_{2} \circ \phi_{2}\right) \cdot \psi\right)+\frac{1}{\gamma} D \phi_{2}: D \psi \mathrm{d} x \\
= & -\int_{\Omega} \frac{1}{\gamma \delta}\left(u_{2} \circ \phi_{2}-u_{1}\right)\left(\left(\nabla u_{2} \circ \phi_{2}\right) \cdot \psi\right)-\frac{1}{\gamma} \Delta \phi_{2} \cdot \psi \mathrm{d} x=: \int_{\Omega} f \cdot \psi \mathrm{d} x
\end{aligned}
$$

with $f \in L^{2}\left(\Omega, \mathbb{R}^{n}\right)$. Then the assertion follows from the general $L^{2}$-regularity theory for polyharmonic equations as presented in [11, section 2.5.2].

Remark 3.3. Since $\phi_{2}$ is a diffeomorphism, (3.10) is equivalent to

$$
u_{2}=\left(\frac{u_{1}-u_{0} \circ \phi_{1}^{-1}}{\operatorname{det}\left(D \phi_{1}\right) \circ \phi_{1}^{-1}}\right) \circ \phi_{2}^{-1}+u_{1} \circ \phi_{2}^{-1} .
$$

Here the first summand reflects the intensity modulation along the geodesic and the second summand quantifies the contribution due to the transport.

We will use the first reformulation (3.7) (Lemma 3.1(i)) of the Euler-Lagrange equation (3.6) w.r.t. $\phi_{2}$ to derive a fixed point iteration in the existence proof for the time discrete exponential map. The second reformulation (3.8) (Lemma 3.1(ii)) will later be used in a modified and spatially discrete fixed point iteration in the numerical algorithm.

4. Local existence and uniqueness of the discrete exponential map. In this section, we prove local existence and local uniqueness for the discrete exponential map. At first, we make use of an argument based on Banach's fixed point theorem applied to the reformulation of the Euler-Lagrange equation given in Lemma 3.1(i) for a discrete geodesic $\left(u_{0}, u_{1}, u_{2}\right)$ with 
deformations $\phi_{1}$ and $\phi_{2}$. For image pairs $\left(u_{0}, u_{1}\right)$ we establish the existence of a solution $\left(u_{2}, \phi_{1}, \phi_{2}\right)$ to the system of equations (3.4), (3.5), and (3.6) provided that $u_{0}$ and $u_{1}$ are bounded in $H^{1}(\Omega)$ and close in $L^{2}(\Omega)$. This does not necessarily imply that for given $u_{0}$ and $u_{1}$ the resulting discrete path $\left(u_{0}, u_{1}, u_{2}\right)$ is the unique discrete geodesic connecting $u_{0}$ and $u_{2}$. Thus, in the second step, we will show that this indeed holds true if the images $u_{0}$ and $u_{1}$ are close in $H^{1}(\Omega)$. To this end, we apply an implicit function theorem argument (cf. the corresponding proof for the discrete exponential map on Hilbert manifolds given in [28]). Let us remark that this argument also allows us to establish existence, but under the stronger assumption that the input images are close in $H^{1}(\Omega)$ compared to the requirement of closeness in $L^{2}(\Omega)$ and boundedness in $H^{1}(\Omega)$ for the existence proof via the fixed point theorem. Furthermore, the fixed point approach will be taken into account for the numerical approximation of the time discrete exponential map.

Theorem 4.1 (existence of solutions of the Euler-Lagrange equations). Let $2 m-\frac{n}{2}>2$, $u_{0} \in H^{1}(\Omega)$. Then there exist constants $C_{u}, c_{u}>0$ such that for every

$$
u_{1} \in\left\{u \in H^{1}(\Omega):|u|_{H^{1}(\Omega)} \leq C_{u},\left\|u-u_{0}\right\|_{L^{2}(\Omega)} \leq c_{u}\right\}
$$

there exists a solution $\left(u_{2}, \phi_{1}, \phi_{2}\right) \in H^{1}(\Omega) \times \mathcal{A} \times \mathcal{A}$ of (3.4), (3.5), and (3.6). In particular, the defining system of equations for $u_{2}=\mathrm{EXP}_{u_{0}}^{2}\left(u_{1}-u_{0}\right)$ is solved.

Proof. We begin with some preparatory considerations.

Let $c_{u} \leq C_{\mathcal{W}}$, and let $\phi_{1} \in \operatorname{argmin}_{\phi \in \mathcal{A}} \mathcal{W}^{D}\left[u_{0}, u_{1}, \phi\right]$ be a minimizing deformation (cf. Proposition 2.1). Following the same line of arguments as for the estimate (2.10) in the proof of Proposition 2.1 we obtain

$$
\left\|\phi_{1}-\mathbb{1}\right\|_{H^{2 m}(\Omega)} \leq C\left\|u_{1}-u_{0}\right\|_{L^{2}(\Omega)} \leq C c_{u} .
$$

Furthermore, taking into account $\mathcal{W}^{D}\left[u_{0}, u_{1}, \phi_{1}\right] \leq \mathcal{W}^{D}\left[u_{0}, u_{1}, \mathbb{1}\right]$ we infer

$$
\left\|u_{1} \circ \phi_{1}-u_{0}\right\|_{L^{2}(\Omega)} \leq \sqrt{\delta \mathcal{W}^{D}\left[u_{0}, u_{1}, \phi_{1}\right]} \leq \sqrt{\delta \mathcal{W}^{D}\left[u_{0}, u_{1}, \mathbb{1}\right]}=\left\|u_{1}-u_{0}\right\|_{L^{2}(\Omega)} \leq c_{u} .
$$

Now we define the fixed point iteration and prove the contraction property in several steps:

(i) Defining the fixed point mapping $\mathcal{F}$. Using Lemma 3.1(i) we define for a fixed deformation $\phi_{1}$ the operators $\mathcal{T}, \mathcal{R}: \mathcal{A} \rightarrow H^{-2 m}(\Omega)$ as

$$
\begin{aligned}
\mathcal{T}[\phi](\psi)= & \int_{\Omega}-\frac{2}{\delta}\left(u_{1} \circ \phi_{1}-u_{0}\right)\left(\nabla u_{1} \cdot(D \phi)^{-1} \psi\right) \circ \phi_{1} \\
& -\frac{1}{\delta} \frac{\left(u_{1} \circ \phi_{1}-u_{0}\right)^{2}}{\operatorname{det} D \phi_{1}}\left((D \phi)^{-T}:\left(D^{2} \phi(D \phi)^{-1} \psi\right)-(D \phi)^{-T}: D \psi\right) \circ \phi_{1} \mathrm{~d} x \\
\mathcal{R}[\phi](\psi)= & \int_{\Omega} 2 \gamma \Delta^{m} \phi \cdot \Delta^{m} \psi+2 D \phi: D \psi \mathrm{d} x
\end{aligned}
$$

for a diffeomorphism $\phi \in \mathcal{A}$ and all $\psi \in H_{0}^{2 m}(\Omega)$. This allows us to reformulate the EulerLagrange equation w.r.t. the deformation $\phi_{2}$ in (3.7) as

$$
\mathcal{T}\left[\phi_{2}\right](\psi)=\mathcal{R}\left[\phi_{2}\right](\psi)
$$

Copyright $\odot$ by SIAM. Unauthorized reproduction of this article is prohibited. 
Next, we will study the invertibility of the linear operator $\mathcal{R}$ and the Lipschitz continuity of $\mathcal{T}$ with a Lipschitz constant which monotonically depends on $c_{u}$ and vanishes for $c_{u} \searrow 0$. This will imply that $\mathcal{F}:=\mathcal{R}^{-1} \circ \mathcal{T}$ is a contraction for sufficiently small $c_{u}$. The fixed point iteration to compute the unknown deformation $\phi_{2}$ reads as $\phi^{j+1}=\mathcal{F}\left[\phi^{j}\right]$ for $j \in \mathbb{N}$ and $\phi^{0}=\mathbb{1}$.

(ii) Lipschitz continuity of $\mathcal{T}$. In what follows, we assume that

$$
\phi, \tilde{\phi} \in B_{\epsilon}(\mathbb{1}):=\left\{\phi: \phi-\mathbb{1} \in H_{0}^{2 m}(\Omega),\|\phi-\mathbb{1}\|_{H^{2 m}(\Omega)}<\epsilon\right\}
$$

for a sufficiently small $\epsilon>0$; the dependency of $\epsilon$ on $C_{u}$ and $c_{u}$ is discussed below. By the embedding $H^{2 m}(\Omega) \hookrightarrow C^{2}(\bar{\Omega})$ and for $\epsilon$ sufficiently small we may assume that

$$
\|D \phi-\mathbb{1}\|_{L^{\infty}(\Omega)}<\frac{1}{2}, \quad\|\operatorname{det}(D \phi)-1\|_{L^{\infty}(\Omega)}<\frac{1}{2}
$$

for all deformations $\phi$ considered. Since $\operatorname{det}(D \phi(x)) \geq \frac{1}{2}$ for all $x \in \Omega$, this ensures that such deformations are in $\mathcal{A}$ and $C^{1}(\Omega)$-diffeomorphisms (see [7, Theorem 5.5-2]). Furthermore, we obtain

$$
\begin{aligned}
\left\|\operatorname{det}\left(D \phi^{-1}\right)\right\|_{L^{\infty}(\Omega)} & \leq\left(1-\|\operatorname{det}(D \phi)-1\|_{L^{\infty}(\Omega)}\right)^{-1}<2, \\
\|\operatorname{cof}(D \phi)\|_{L^{\infty}(\Omega)} & \leq C, \quad\left\|(D \phi)^{-1}\right\|_{L^{\infty}(\Omega)} \leq C
\end{aligned}
$$

and deduce from $(D \phi)^{-1}=(\operatorname{det}(D \phi))^{-1} \operatorname{cof}(D \phi)^{T}$

$$
\begin{aligned}
& \left\|(D \phi)^{-1}-(D \tilde{\phi})^{-1}\right\|_{L^{\infty}(\Omega)}=\left\|(\operatorname{det}(D \phi))^{-1} \operatorname{cof}(D \phi)^{T}-(\operatorname{det}(D \tilde{\phi}))^{-1} \operatorname{cof}(D \tilde{\phi})^{T}\right\|_{L^{\infty}(\Omega)} \\
\leq & \left\|\frac{(\operatorname{cof}(D \phi))^{T}}{\operatorname{det}(D \phi) \operatorname{det}(D \tilde{\phi})}\right\|_{L^{\infty}(\Omega)}\|\operatorname{det}(D \phi)-\operatorname{det}(D \tilde{\phi})\|_{L^{\infty}(\Omega)} \\
& +\left\|(\operatorname{det}(D \tilde{\phi}))^{-1}\right\|_{L^{\infty}(\Omega)}\left\|\operatorname{cof}(D \phi)^{T}-\operatorname{cof}(D \tilde{\phi})^{T}\right\|_{L^{\infty}(\Omega)} \\
\leq & C\|\phi-\tilde{\phi}\|_{H^{2 m}(\Omega)}
\end{aligned}
$$

for deformations $\phi, \tilde{\phi} \in B_{\epsilon}(\mathbb{1})$. Thus, using the Cauchy-Schwarz inequality, the transformation formula, (4.2), (4.3), and (4.4) we achieve the following estimate corresponding to the first term of $\mathcal{T}$ :

$$
\begin{aligned}
& \mid \int_{\Omega}\left(u_{1} \circ \phi_{1}-u_{0}\right)\left(\nabla u_{1} \circ \phi_{1}\right) \cdot\left((D \phi)^{-1} \psi\right) \circ \phi_{1} \\
& \quad-\left(u_{1} \circ \phi_{1}-u_{0}\right)\left(\nabla u_{1} \circ \phi_{1}\right) \cdot\left((D \tilde{\phi})^{-1} \psi\right) \circ \phi_{1} \mathrm{~d} x \mid \\
\leq & C\left\|u_{1} \circ \phi_{1}-u_{0}\right\|_{L^{2}(\Omega)}\left|u_{1}\right|_{H^{1}(\Omega)}\left\|\operatorname{det} D\left(\phi_{1}^{-1}\right)\right\|_{L^{\infty}(\Omega)}\left\|(D \phi)^{-1}-(D \tilde{\phi})^{-1}\right\|_{L^{\infty}(\Omega)}\|\psi\|_{L^{\infty}(\Omega)} \\
\leq & C C_{u} c_{u}\|\phi-\tilde{\phi}\|_{H^{2 m}(\Omega)}\|\psi\|_{H^{2 m}(\Omega)} .
\end{aligned}
$$

Likewise, for the second term of $\mathcal{T}$ we obtain by the transformation formula and by the embedding $H^{2 m}(\Omega) \hookrightarrow C^{2}(\bar{\Omega})$

$$
\mid \int_{\Omega} \frac{\left(u_{1} \circ \phi_{1}-u_{0}\right)^{2}}{\operatorname{det} D \phi_{1}}\left((D \phi)^{-T}:\left(D^{2} \phi(D \phi)^{-1} \psi\right)-(D \tilde{\phi})^{-T}:\left(D^{2} \tilde{\phi}(D \tilde{\phi})^{-1} \psi\right)\right.
$$




$$
\begin{gathered}
\left.-(D \phi)^{-T}: D \psi+(D \tilde{\phi})^{-T}: D \psi\right)\left.\circ \phi_{1} \mathrm{~d} x\right|^{\leq} \\
\leq C c_{u}^{2}\left\|\left(\operatorname{det} D \phi_{1}\right)^{-1}\right\|_{L^{\infty}(\Omega)}( \\
+\left\|(D \phi)^{-T}:\left(D^{2} \phi(D \phi)^{-1} \psi\right)-(D \tilde{\phi})^{-T}:\left(D^{2} \tilde{\phi}(D \tilde{\phi})^{-1} \psi\right)\right\|_{L^{\infty}(\Omega)} \\
\left.+\left\|(D \phi)^{-T}: D \psi-(D \tilde{\phi})^{-T}: D \psi\right\|_{L^{\infty}(\Omega)}\right) \\
\leq C c_{u}^{2}\|\phi-\tilde{\phi}\|_{H^{2 m}(\Omega)}\|\psi\|_{H^{2 m}(\Omega)} .
\end{gathered}
$$

To conclude, for $\left\|u_{1}-u_{0}\right\|_{L^{2}(\Omega)} \leq c_{u}$ and $\left|u_{1}\right|_{H^{1}(\Omega)} \leq C_{u}$ the mapping $\mathcal{T}$ is indeed Lipschitz continuous on $B_{\epsilon}(\mathbb{1}) \subset \mathcal{A}$ and the Lipschitz constant is bounded by $C\left(C_{u} c_{u}+c_{u}^{2}\right)$.

(iii) Invertibility of $\mathcal{R}$. The bilinear form

$$
\widetilde{\mathcal{R}}: H_{0}^{2 m}(\Omega) \times H_{0}^{2 m}(\Omega) \rightarrow \mathbb{R}, \quad(\zeta, \psi) \mapsto \int_{\Omega} 2 \gamma \Delta^{m} \zeta \cdot \Delta^{m} \psi+2 D \zeta: D \psi \mathrm{d} x
$$

is bounded in $H_{0}^{2 m}(\Omega)$. Furthermore, $\widetilde{\mathcal{R}}$ is coercive since for any $\psi \in H_{0}^{2 m}(\Omega)$ we obtain

$$
\|\psi\|_{H^{2 m}(\Omega)}^{2} \leq C|\psi|_{H^{2 m}(\Omega)}^{2}=C \int_{\Omega} \Delta^{m} \psi \cdot \Delta^{m} \psi \mathrm{d} x
$$

due to (2.7) and the iterative application of the Poincaré inequality (cf. [1, Corollary 6.31]). Hence, by the Lax-Milgram theorem (cf. [12]) there exists for each $z \in H^{-2 m}(\Omega)$ a unique $\zeta \in H_{0}^{2 m}(\Omega)$ such that $\widetilde{\mathcal{R}}[\zeta](\psi)=z(\psi)$ and $\widetilde{\mathcal{R}}^{-1}: H^{-2 m}(\Omega) \rightarrow H_{0}^{2 m}(\Omega)$ is a bounded operator. Finally, since $\mathcal{R}[\phi]=\widetilde{\mathcal{R}}[\phi-\mathbb{1}]$, we can infer that $\mathcal{R}$ is a bounded and invertible operator with inverse $\mathcal{R}^{-1}[z]=\mathbb{1}+\widetilde{\mathcal{R}}^{-1}[z]$.

(iv) Contraction property of $\mathcal{F}$. Using the boundedness of $\mathcal{R}^{-1}$ and the Lipschitz continuity of $\mathcal{T}$ we obtain for $\mathcal{F}:=\mathcal{R}^{-1} \circ \mathcal{T}$

$$
\|\mathcal{F}[\phi]-\mathcal{F}[\tilde{\phi}]\|_{H^{2 m}(\Omega)} \leq C\|\mathcal{T}[\phi]-\mathcal{T}[\tilde{\phi}]\|_{H^{-2 m}(\Omega)} \leq C\left(C_{u} c_{u}+c_{u}^{2}\right)\|\phi-\tilde{\phi}\|_{H^{2 m}(\Omega)}
$$

for $\phi, \tilde{\phi} \in B_{\epsilon}(\mathbb{1})$, which proves that $\mathcal{F}$ is contractive for sufficiently small $C_{u}, c_{u}$, and $\epsilon$.

Next, we prove $\mathcal{F}: B_{\epsilon}(\mathbb{1}) \rightarrow B_{\epsilon}(\mathbb{1})$ for proper choices of $C_{u}, c_{u}$, and $\epsilon$. By using the boundedness of $\mathcal{R}^{-1}$ and $\mathcal{R}[\mathbb{1}]=0$ one can infer

$\|\mathcal{F}[\mathbb{1}]-\mathbb{1}\|_{H^{2 m}(\Omega)}=\left\|\mathcal{R}^{-1} \circ \mathcal{T}[\mathbb{1}]-\mathcal{R}^{-1} \circ \mathcal{R}[\mathbb{1}]\right\|_{H^{2 m}(\Omega)} \leq C\|(\mathcal{T}-\mathcal{R})[\mathbb{1}]\|_{H^{-2 m}(\Omega)} \leq C\left(C_{u} c_{u}+c_{u}^{2}\right)$.

Thus, for any $\phi \in B_{\epsilon}(\mathbb{1})$ one gets

$$
\begin{aligned}
& \|\mathcal{F}[\phi]-\mathbb{1}\|_{H^{2 m}(\Omega)} \leq\|\mathcal{F}[\phi]-\mathcal{F}[\mathbb{1}]\|_{H^{2 m}(\Omega)}+\|\mathcal{F}[\mathbb{1}]-\mathbb{1}\|_{H^{2 m}(\Omega)} \\
\leq & C\left(C_{u} c_{u}+c_{u}^{2}\right)\|\phi-\mathbb{1}\|_{H^{2 m}(\Omega)}+C\left(C_{u} c_{u}+c_{u}^{2}\right) \leq C\left(C_{u} c_{u}+c_{u}^{2}\right) \epsilon+C\left(C_{u} c_{u}+c_{u}^{2}\right) .
\end{aligned}
$$

Now, choosing $C_{u}, c_{u}$ small enough and $\epsilon$ such that the conditions in (4.3) are satisfied for any $\phi \in B_{\epsilon}(\mathbb{1})$ and for $\phi_{1}, \mathcal{F}$ maps $B_{\epsilon}(\mathbb{1})$ onto $B_{\epsilon}(\mathbb{1})$.

Hence, the application of Banach's fixed point theorem proves the existence of a unique deformation $\phi_{2}$ in $B_{\epsilon}(\mathbb{1}) \subset \mathcal{A}$ solving (3.7). Then the unique image $u_{2}$ associated with $\left(\phi_{1}, \phi_{2}\right)$ can be computed using the formula (3.11). Thus, there exists a solution $\left(u_{2}, \phi_{1}, \phi_{2}\right) \in$ $H^{1}(\Omega) \times \mathcal{A} \times \mathcal{A}$ of (3.4), (3.5), and (3.6), and this solution is unique in a small neighborhood of $\left(u_{0}, \mathbb{1}, \mathbb{1}\right)$. 
Theorem 4.2 (local uniqueness and well-posedness of the discrete exponential map). Let $2 m-\frac{n}{2}>2$ and $u_{0} \in H^{1}(\Omega)$. Then there exist neighborhoods $\mathbf{U} \subset H^{1}(\Omega)$ of $u_{0}$ and $\mathbf{D} \subset \mathcal{A}$ of $\mathbb{1}$ such that for every $u_{2} \in \mathbf{U}$ there exists at most one solution $\left(u_{1}, \phi_{1}, \phi_{2}\right) \in \mathbf{U} \times \mathbf{D} \times \mathbf{D}$ of (3.4)-(3.6). In particular, the discrete exponential map is locally well-posed and

$$
u_{2}=\operatorname{EXP}_{u_{0}}^{2}\left(u_{1}-u_{0}\right)
$$

Proof. At first, we want to get rid of the unknown image $u_{1}$. To this end, we observe that the sum of the two matching terms in $\mathcal{W}^{D}\left[u_{0}, u_{1}, \phi_{1}\right]+\mathcal{W}^{D}\left[u_{1}, u_{2}, \phi_{2}\right]$ can be rewritten as follows:

$$
\int_{\Omega}\left(u_{1} \circ \phi_{1}-u_{0}\right)^{2}+\left(u_{2} \circ \phi_{2}-u_{1}\right)^{2} \mathrm{~d} x=\int_{\Omega}\left(u_{1} \circ \phi_{1}-u_{0}\right)^{2}+\left(u_{2} \circ \phi_{2} \circ \phi_{1}-u_{1} \circ \phi_{1}\right)^{2} \operatorname{det} D \phi_{1} \mathrm{~d} x \text {. }
$$

Therefore, the image $u_{1}$ minimizing the above integral is characterized pointwise a.e. on $\Omega$ by

$$
u_{1} \circ \phi_{1}=\frac{u_{0}+\left(u_{2} \circ \phi_{2} \circ \phi_{1}\right) \operatorname{det} D \phi_{1}}{1+\operatorname{det} D \phi_{1}}
$$

and can thus be written as a function $u_{1}\left(u_{2}, \phi_{1}, \phi_{2}\right)$ of the image $u_{2}$ and the deformations $\phi_{1}$ and $\phi_{2}$ (we omit the dependence on the image $u_{0}$ ). Hence, the Euler-Lagrange equations (3.4)-(3.6) can be reformulated as

$$
0=\mathcal{K}\left[u_{2}, \phi_{1}, \phi_{2}\right]:=\partial_{\left(\phi_{1}, \phi_{2}\right)} \mathcal{J}\left[u_{2}, \phi_{1}, \phi_{2}\right],
$$

where $\mathcal{J}$ is a functional on $H^{1}(\Omega) \times \mathcal{A} \times \mathcal{A}$ with

$$
\begin{gathered}
\mathcal{J}\left[u_{2}, \phi_{1}, \phi_{2}\right]=\mathcal{W}^{D}\left[u_{0}, u_{1}\left(u_{2}, \phi_{1}, \phi_{2}\right), \phi_{1}\right]+\mathcal{W}^{D}\left[u_{1}\left(u_{2}, \phi_{1}, \phi_{2}\right), u_{2}, \phi_{2}\right] \\
=\int_{\Omega}\left|D \phi_{1}-\mathbb{1}\right|^{2}+\gamma\left|\Delta^{m} \phi_{1}\right|^{2}+\left|D \phi_{2}-\mathbb{1}\right|^{2}+\gamma\left|\Delta^{m} \phi_{2}\right|^{2} \\
\quad+\frac{h\left(D \phi_{1}\right)}{\delta}\left(u_{2} \circ \phi_{2} \circ \phi_{1}-u_{0}\right)^{2} \mathrm{~d} x
\end{gathered}
$$

for $h(A)=\frac{\operatorname{det} A}{1+\operatorname{det} A}$ and $\mathcal{K}: H^{1}(\Omega) \times \mathcal{A} \times \mathcal{A} \rightarrow\left(H_{0}^{2 m}(\Omega) \times H_{0}^{2 m}(\Omega)\right)^{\prime}$. Now we will show that in a neighborhood of $\left(u_{0}, \mathbb{1}, \mathbb{1}\right)$ one obtains an explicit representation $\left(\phi_{1}, \phi_{2}\right)\left[u_{2}\right]$ for the implicit equation $0=\mathcal{K}\left[u_{2}, \phi_{1}, \phi_{2}\right]$ via the implicit function theorem. Hence, for every $u_{2}$, which is close to $u_{0}$ in $H^{1}(\Omega)$, there exists in a small neighborhood of $\mathcal{A} \times \mathcal{A}$ a unique tuple $\left(\phi_{1}, \phi_{2}\right)$, which solves the above implicit equation. This indeed proves the claim. To apply the implicit function theorem, we have to show that $\partial_{\left(\phi_{1}, \phi_{2}\right)} \mathcal{K}\left[u_{0}, \mathbb{1}, \mathbb{1}\right]=\partial_{\left(\phi_{1}, \phi_{2}\right)}^{2} \mathcal{J}\left[u_{0}, \mathbb{1}, \mathbb{1}\right]$ is invertible with bounded inverse. At first, we compute all components of $\partial_{\left(\phi_{1}, \phi_{2}\right)}^{2} \mathcal{J}\left[u_{2}, \phi_{1}, \phi_{2}\right]$. For this reason we focus here on the variation of

$$
\widetilde{\mathcal{J}}\left[u_{2}, \phi_{1}, \phi_{2}\right]=\int_{\Omega} h\left(D \phi_{1}\right)\left(u_{2} \circ \phi_{2} \circ \phi_{1}-u_{0}\right)^{2} \mathrm{~d} x ;
$$

the derivatives of the other components of $\mathcal{J}$ are straightforward. We use integration by parts to avoid derivatives of the involved image intensities. For the first variation w.r.t. $\phi_{1}$ and $\phi_{2}$ we obtain

$$
\partial_{\phi_{1}} \widetilde{\mathcal{J}}\left[u_{2}, \phi_{1}, \phi_{2}\right](\psi)=\int_{\Omega}\left(u_{2} \circ \phi_{2} \circ \phi_{1}-u_{0}\right)^{2} D h\left(D \phi_{1}\right): D \psi
$$

Copyright (C) by SIAM. Unauthorized reproduction of this article is prohibited. 


$$
\begin{gathered}
+2 h\left(D \phi_{1}\right)\left(u_{2} \circ \phi_{2} \circ \phi_{1}-u_{0}\right) \nabla\left(u_{2} \circ \phi_{2} \circ \phi_{1}\right) \cdot\left(D \phi_{1}\right)^{-1} \psi \mathrm{d} x \\
=\int_{\Omega}\left(u_{2} \circ \phi_{2} \circ \phi_{1}-u_{0}\right)^{2} D h\left(D \phi_{1}\right): D \psi \\
\quad-\left(u_{2} \circ \phi_{2} \circ \phi_{1}\right)^{2} \operatorname{div}\left(h\left(D \phi_{1}\right)\left(D \phi_{1}\right)^{-1} \psi\right) \\
\quad+2\left(u_{2} \circ \phi_{2} \circ \phi_{1}\right) \operatorname{div}\left(u_{0} h\left(D \phi_{1}\right)\left(D \phi_{1}\right)^{-1} \psi\right) \mathrm{d} x, \\
\partial_{\phi_{2}} \tilde{\mathcal{J}}\left[u_{2}, \phi_{1}, \phi_{2}\right](\psi)=\int_{\Omega} 2 h\left(D \phi_{1}\right)\left(u_{2} \circ \phi_{2} \circ \phi_{1}-u_{0}\right) \\
\nabla\left(u_{2} \circ \phi_{2} \circ \phi_{1}\right) \cdot\left(D\left(\phi_{2} \circ \phi_{1}\right)\right)^{-1}\left(\psi \circ \phi_{1}\right) \mathrm{d} x \\
=\int_{\Omega} 2\left(u_{2} \circ \phi_{2} \circ \phi_{1}\right) \operatorname{div}\left(u_{0} h\left(D \phi_{1}\right)\left(D\left(\phi_{2} \circ \phi_{1}\right)\right)^{-1}\left(\psi \circ \phi_{1}\right)\right) \\
-\left(u_{2} \circ \phi_{2} \circ \phi_{1}\right)^{2} \operatorname{div}\left(h\left(D \phi_{1}\right)\left(D\left(\phi_{2} \circ \phi_{1}\right)\right)^{-1}\left(\psi \circ \phi_{1}\right)\right) \mathrm{d} x
\end{gathered}
$$

using the following different versions of the chain rule:

$$
\begin{aligned}
\left(\nabla\left(u_{2} \circ \phi_{2} \circ \phi_{1}\right)\right)^{T} & =\left(\nabla\left(u_{2} \circ \phi_{2}\right) \circ \phi_{1}\right)^{T} D \phi_{1}, \\
\nabla\left(u_{2} \circ \phi_{2} \circ \phi_{1}\right)^{2} & =2\left(u_{2} \circ \phi_{2} \circ \phi_{1}\right) \nabla\left(u_{2} \circ \phi_{2} \circ \phi_{1}\right), \\
\left(\nabla\left(u_{2} \circ \phi_{2} \circ \phi_{1}\right)\right)^{T} & =\left(\nabla u_{2} \circ\left(\phi_{2} \circ \phi_{1}\right)\right)^{T} D\left(\phi_{2} \circ \phi_{1}\right) .
\end{aligned}
$$

Then for the second order variations one gets

$$
\begin{aligned}
& \partial_{\phi_{1}}^{2} \widetilde{\mathcal{J}}\left[u_{2}, \phi_{1}, \phi_{2}\right](\psi, \zeta) \\
& =\int_{\Omega} 2\left(u_{2} \circ \phi_{2} \circ \phi_{1}-u_{0}\right) \nabla\left(u_{2} \circ \phi_{2}\right) \circ \phi_{1} \cdot \zeta\left(D h\left(D \phi_{1}\right): D \psi\right) \\
& \quad+\left(u_{2} \circ \phi_{2} \circ \phi_{1}-u_{0}\right)^{2} D^{2} h\left(D \phi_{1}\right)(D \psi, D \zeta) \\
& \quad-2\left(u_{2} \circ \phi_{2} \circ \phi_{1}\right) \nabla\left(u_{2} \circ \phi_{2}\right) \circ \phi_{1} \cdot \zeta \operatorname{div}\left(h\left(D \phi_{1}\right)\left(D \phi_{1}\right)^{-1} \psi\right) \\
& \quad-\left(u_{2} \circ \phi_{2} \circ \phi_{1}\right)^{2} \operatorname{div}\left(\partial_{\phi_{1}}\left(h\left(D \phi_{1}\right)\left(D \phi_{1}\right)^{-1}\right)(\zeta) \psi\right) \\
& \quad+2 \nabla\left(u_{2} \circ \phi_{2}\right) \circ \phi_{1} \cdot \zeta \operatorname{div}\left(u_{0} h\left(D \phi_{1}\right)\left(D \phi_{1}\right)^{-1} \psi\right) \\
& \quad+2\left(u_{2} \circ \phi_{2} \circ \phi_{1}\right) \operatorname{div}\left(u_{0} \partial_{\phi_{1}}\left(h\left(D \phi_{1}\right)\left(D \phi_{1}\right)^{-1}\right)(\zeta) \psi\right) \mathrm{d} x, \\
& \partial_{\phi_{2}}^{2} \widetilde{\mathcal{J}}\left[u_{2}, \phi_{1}, \phi_{2}\right](\psi, \zeta) \\
& \int_{\Omega} 2 \nabla u_{2} \circ\left(\phi_{2} \circ \phi_{1}\right) \cdot\left(\zeta \circ \phi_{1}\right) \operatorname{div}\left(u_{0} h\left(D \phi_{1}\right)\left(D\left(\phi_{2} \circ \phi_{1}\right)\right)^{-1}\left(\psi \circ \phi_{1}\right)\right) \\
& \quad+2\left(u_{2} \circ \phi_{2} \circ \phi_{1}\right) \operatorname{div}\left(u_{0} h\left(D \phi_{1}\right) \partial_{\phi_{2}}\left(\left(D\left(\phi_{2} \circ \phi_{1}\right)\right)^{-1}\right)(\zeta)\left(\psi \circ \phi_{1}\right)\right) \\
& \quad-2\left(u_{2} \circ \phi_{2} \circ \phi_{1}\right) \nabla u_{2} \circ\left(\phi_{2} \circ \phi_{1}\right) \cdot\left(\zeta \circ \phi_{1}\right) \operatorname{div}\left(h\left(D \phi_{1}\right)\left(D\left(\phi_{2} \circ \phi_{1}\right)\right)^{-1}\left(\psi \circ \phi_{1}\right)\right) \\
& \quad-\left(u_{2} \circ \phi_{2} \circ \phi_{1}\right)^{2} \operatorname{div}\left(h\left(D \phi_{1}\right) \partial_{\phi_{2}}\left(\left(D\left(\phi_{2} \circ \phi_{1}\right)\right)^{-1}\right)(\zeta)\left(\psi \circ \phi_{1}\right)\right) \mathrm{d} x, \\
& \partial_{\phi_{1}} \partial_{\phi_{2}} \widetilde{\mathcal{J}}\left[u_{2}, \phi_{1}, \phi_{2}\right](\psi, \zeta) \\
& =\int_{\Omega} 2 \nabla\left(u_{2} \circ \phi_{2}\right) \circ \phi_{1} \cdot \psi \operatorname{div}\left(u_{0} h\left(D \phi_{1}\right)\left(D\left(\phi_{2} \circ \phi_{1}\right)\right)^{-1}\left(\zeta \circ \phi_{1}\right)\right) \\
& \quad+2\left(u_{2} \circ \phi_{2} \circ \phi_{1}\right) \operatorname{div}\left(u_{0} \partial_{\phi_{1}}\left(h\left(D \phi_{1}\right)\left(D\left(\phi_{2} \circ \phi_{1}\right)\right)^{-1}\left(\zeta \circ \phi_{1}\right)\right)(\psi)\right) \\
& \quad-2\left(u_{2} \circ \phi_{2} \circ \phi_{1}\right) \nabla\left(u_{2} \circ \phi_{2}\right) \circ \phi_{1} \cdot \psi \operatorname{div}\left(h\left(D \phi_{1}\right)\left(D\left(\phi_{2} \circ \phi_{1}\right)\right)^{-1}\left(\zeta \circ \phi_{1}\right)\right) \\
& \quad-\left(u_{2} \circ \phi_{2} \circ \phi_{1}\right)^{2} \operatorname{div}\left(\partial_{\phi_{1}}\left(h\left(D \phi_{1}\right)\left(D\left(\phi_{2} \circ \phi_{1}\right)\right)^{-1}\left(\zeta \circ \phi_{1}\right)\right)(\psi)\right) \mathrm{d} x .
\end{aligned}
$$

Evaluating the second order variational derivatives at the point $\left(u_{0}, \mathbb{1}, \mathbb{1}\right)$ yields

$$
\partial_{\phi_{1}}^{2} \tilde{\mathcal{J}}\left[u_{0}, \mathbb{1}, \mathbb{1}\right](\psi, \zeta)
$$




$$
\begin{aligned}
= & \int_{\Omega}-u_{0} \nabla u_{0} \cdot \zeta \operatorname{div}(\psi)-u_{0}^{2} \operatorname{div}\left(\left.\partial_{\phi_{1}}\left(h\left(D \phi_{1}\right)\left(D \phi_{1}\right)^{-1}\right)\right|_{\phi_{1}=\mathbb{1}}(\zeta) \psi\right) \\
& +\nabla u_{0} \cdot \zeta \operatorname{div}\left(u_{0} \psi\right)+2 u_{0} \operatorname{div}\left(\left.u_{0} \partial_{\phi_{1}}\left(h\left(D \phi_{1}\right)\left(D \phi_{1}\right)^{-1}\right)\right|_{\phi_{1}=\mathbb{1}}(\zeta) \psi\right) \mathrm{d} x \\
= & \int_{\Omega} \zeta^{T} \nabla u_{0} \nabla u_{0}^{T} \psi \mathrm{d} x, \\
& \partial_{\phi_{2}}^{2} \widetilde{\mathcal{J}}\left[u_{0}, \mathbb{1}, \mathbb{1}\right](\psi, \zeta) \\
= & \int_{\Omega} \nabla u_{0} \cdot \zeta \operatorname{div}\left(u_{0} \psi\right)+u_{0} \operatorname{div}\left(\left.u_{0} \partial_{\phi_{2}}\left(\left(D\left(\phi_{2} \circ \phi_{1}\right)\right)^{-1}\right)\right|_{\phi_{2}=\mathbb{1}}(\zeta)\left(\psi \circ \phi_{1}\right)\right) \\
& -u_{0} \nabla u_{0} \cdot \zeta \operatorname{div}(\psi)-\frac{1}{2} u_{0}^{2} \operatorname{div}\left(\left.\partial_{\phi_{2}}\left(\left(D\left(\phi_{2} \circ \phi_{1}\right)\right)^{-1}\right)\right|_{\phi_{2}=\mathbb{1}}(\zeta)\left(\psi \circ \phi_{1}\right)\right) \mathrm{d} x \\
& \int_{\Omega} \zeta^{T} \nabla u_{0} \nabla u_{0}^{T} \psi \mathrm{d} x, \\
& \partial_{\phi_{1}} \partial_{\phi_{2}} \widetilde{\mathcal{J}}\left[u_{0}, \mathbb{1}, \mathbb{1}\right](\psi, \zeta) \\
= & \int_{\Omega} \nabla u_{0} \cdot \psi \operatorname{div}\left(u_{0} \zeta\right)+2 u_{0} \operatorname{div}\left(\left.u_{0} \partial_{\phi_{1}}\left(h\left(D \phi_{1}\right)\left(D\left(\phi_{2} \circ \phi_{1}\right)\right)^{-1}\left(\zeta \circ \phi_{1}\right)\right)\right|_{\phi_{1}=\mathbb{1}}(\psi)\right) \\
& -u_{0} \nabla u_{0} \cdot \psi \operatorname{div}(\zeta)-u_{0}^{2} \operatorname{div}\left(\left.\partial_{\phi_{1}}\left(h\left(D \phi_{1}\right)\left(D\left(\phi_{2} \circ \phi_{1}\right)\right)^{-1}\left(\zeta \circ \phi_{1}\right)\right)\right|_{\phi_{1}=\mathbb{1}}(\psi)\right) \mathrm{d} x \\
= & \int_{\Omega} \zeta^{T} \nabla u_{0} \nabla u_{0}^{T} \psi \mathrm{d} x .
\end{aligned}
$$

Here we have used the following identities, which rely on integration by parts:

$$
\begin{aligned}
\int_{\Omega} u_{0}^{2} \operatorname{div} v-2 u_{0} \operatorname{div}\left(u_{0} v\right) \mathrm{d} x & =\int_{\Omega}-\nabla u_{0}^{2} \cdot v-2 u_{0} \operatorname{div}\left(u_{0} v\right) \mathrm{d} x \\
& =\int_{\Omega}-2 \nabla u_{0} \cdot\left(u_{0} v\right)-2 u_{0} \operatorname{div}\left(u_{0} v\right) \mathrm{d} x \\
& =\int_{\Omega} 2 u_{0} \operatorname{div}\left(u_{0} v\right)-2 u_{0} \operatorname{div}\left(u_{0} v\right) \mathrm{d} x=0
\end{aligned}
$$

for any vector field $v \in H_{0}^{1}\left(\Omega, \mathbb{R}^{n}\right)$. Altogether, also taking into account the second order variation of the remaining terms of $\mathcal{J}$ we obtain

$$
\begin{array}{r}
\partial_{\left(\phi_{1}, \phi_{2}\right)}^{2} \mathcal{J}\left[u_{0}, \mathbb{1}, \mathbb{1}\right]\left(\left(\psi_{1}, \psi_{2}\right),\left(\zeta_{1}, \zeta_{2}\right)\right)=\int_{\Omega} 2 \gamma \Delta^{m} \psi_{1} \cdot \Delta^{m} \zeta_{1}+2 D \psi_{1}: D \zeta_{1}+2 \gamma \Delta^{m} \psi_{2} \cdot \Delta^{m} \zeta_{2} \\
+2 D \psi_{2}: D \zeta_{2}+\frac{1}{\delta}\left(\psi_{1}+\psi_{2}\right)^{T} \nabla u_{0} \nabla u_{0}^{T}\left(\zeta_{1}+\zeta_{2}\right) \mathrm{d} x
\end{array}
$$

It is straightforward to verify that $\partial_{\left(\phi_{1}, \phi_{2}\right)}^{2} \mathcal{J}\left[u_{0}, \mathbb{1}, \mathbb{1}\right]$ is a continuous bilinear form on $H_{0}^{2 m}(\Omega) \times$ $H_{0}^{2 m}(\Omega)$ by taking into account the estimate

$$
\left|\int_{\Omega}\left(\psi_{1}+\psi_{2}\right)^{T} \nabla u_{0} \nabla u_{0}^{T}\left(\zeta_{1}+\zeta_{2}\right) \mathrm{d} x\right| \leq C\left\|u_{0}\right\|_{H^{1}(\Omega)}^{2}\left\|\left(\psi_{1}, \psi_{2}\right)\right\|_{H^{2 m}(\Omega)}\left\|\left(\zeta_{1}, \zeta_{2}\right)\right\|_{H^{2 m}(\Omega)} ;
$$

the coercivity follows by arguments analogous to those in the proof of the coercivity of $\mathcal{R}$ (cf. Theorem 4.1). Thus, the Lax-Milgram theorem ensures the required invertibility. 
5. Spatial discretization and fixed point algorithm. In what follows, we introduce a spatial discretization scheme as well as an algorithm to compute the discrete exponential map based on the time discrete operator $\operatorname{EXP}_{u_{0}}^{2}\left(u_{1}-u_{0}\right)$ for given images $u_{0}$ and $u_{1}$.

Let us recall that the computation of $\mathrm{EXP}^{k}$ for $k>2$ requires the iterative application of $\mathrm{EXP}^{2}$ as defined in (3.1). Explicitly, we ask for a numerical approximation of the matching deformations $\phi_{1}, \phi_{2}$ and the actual succeeding image $u_{2}=\operatorname{EXP}_{u_{0}}^{2}\left(u_{1}-u_{0}\right)$ along the shot discrete path. Here we restrict ourselves to two-dimensional images and, for the sake of simplicity, we assume that the image domain is the unit square, i.e., $\Omega=(0,1)^{2}$. Conceptually, the generalization to three dimensions is straightforward. As a simplification for the numerical implementation, we restrict ourselves to the case $m=1$ despite the theoretical requirement that $m>1+\frac{n}{4}=\frac{3}{2}$. Below, we will introduce the space of tensor product cubic splines for the discretization of deformations. For such discrete deformations the reformulation in Lemma 3.1(ii) holds true (the regularity result in Proposition 3.2 is only required for the reformulation in the spatially continuous case). We experimentally observed that the spatially discretized model ensures sufficient regularity of the deformations to reliably solve the EulerLagrange equations numerically.

To sum up, the discrete energy density that we will employ in all numerical computations is given by

$$
\mathcal{W}^{D}[u, \tilde{u}, \phi]=\int_{\Omega}|D \phi-\mathbb{1}|^{2}+\gamma \Delta \phi \cdot \Delta \phi+\frac{1}{\delta}(\tilde{u} \circ \phi-u)^{2} \mathrm{~d} x
$$

for $u, \tilde{u} \in H^{1}(\Omega)$ and $\phi \in \mathcal{A}$.

The algorithm to compute $\phi_{2}$ is based on a spatially discrete fixed point iteration similar to the one used in the proof of Theorem 4.1. Explicitly, we follow the derivation of the fixed point mapping in this proof now using the reformulation (ii) instead of (i) in Lemma 3.1 as a starting point and define

$$
\begin{aligned}
\widetilde{\mathcal{T}}[\phi](\psi)= & \int_{\Omega} 2 \gamma \Delta \phi_{1} \cdot \Delta\left(\left((D \phi)^{-1} \psi\right) \circ \phi_{1}\right)+2 D \phi_{1}: D\left(\left((D \phi)^{-1} \psi\right) \circ \phi_{1}\right) \\
& -\frac{1}{\delta} \frac{\left(u_{1} \circ \phi_{1}-u_{0}\right)^{2}}{\operatorname{det} D \phi_{1}}\left((D \phi)^{-T}:\left(D^{2} \phi(D \phi)^{-1} \psi\right)-(D \phi)^{-T}: D \psi\right) \circ \phi_{1} \mathrm{~d} x \\
= & \int_{\Omega}-2 \gamma D \Delta \phi_{1}:\left(D\left((D \phi)^{-1} \psi\right) \circ \phi_{1}\right)-2 \Delta \phi_{1} \cdot\left((D \phi)^{-1} \psi\right) \circ \phi_{1} \\
& -\frac{1}{\delta} \frac{\left(u_{1} \circ \phi_{1}-u_{0}\right)^{2}}{\operatorname{det} D \phi_{1}}\left((D \phi)^{-T}:\left(D^{2} \phi(D \phi)^{-1} \psi\right)-(D \phi)^{-T}: D \psi\right) \circ \phi_{1} \mathrm{~d} x
\end{aligned}
$$

for all $\psi \in H_{0}^{2 m}(\Omega)$. Here we used integration by parts to get the second equality. This ansatz is numerically beneficial because it avoids the evaluation of gradients of image intensities. In fact, we experimentally observed that the evaluation of the expression $\int_{\Omega}\left(u_{1} \circ \phi_{1}-u_{0}\right)\left(\nabla u_{1}\right.$. $\left.(D \phi)^{-1} \psi\right) \circ \phi_{1} \mathrm{~d} x$ appearing in the definition of $\mathcal{T}$ in the proof of Theorem 4.1 suffers from accuracy problems in the proximity of interfaces of $u_{1}$ due to the approximate numerical quadrature. To further improve the stability of the numerical algorithm with respect to the evaluation of the first integrand, we additionally rewrite this expression by making use of

Copyright (C) by SIAM. Unauthorized reproduction of this article is prohibited. 
$A: B=\operatorname{tr}\left(A^{T} B\right)$ as follows:

$$
\begin{aligned}
\left.\int_{\Omega} D \Delta \phi_{1}: D\left((D \phi)^{-1} \psi\right) \circ \phi_{1}\right) \mathrm{d} x= & \int_{\Omega} D \Delta \phi_{1}:\left((D \phi)^{-1} \circ \phi_{1}\right) D\left(\psi \circ \phi_{1}\right) \\
& +D \Delta \phi_{1}: D\left((D \phi)^{-1} \circ \phi_{1}\right)\left(\psi \circ \phi_{1}\right) \mathrm{d} x \\
= & \int_{\Omega}\left((D \phi)^{-T} \circ \phi_{1}\right) D \Delta \phi_{1}: D\left(\psi \circ \phi_{1}\right) \\
& +D \Delta \phi_{1}: D\left((D \phi)^{-1} \circ \phi_{1}\right)\left(\psi \circ \phi_{1}\right) \mathrm{d} x .
\end{aligned}
$$

The second operator $\mathcal{R}$ is chosen identically to the one in the proof of Theorem 4.1. Then, taking into account the identity $\widetilde{\mathcal{T}}\left[\phi_{2}\right](\psi)=\mathcal{R}\left[\phi_{2}\right](\psi)$ for all test functions $\psi \in H_{0}^{2 m}(\Omega)$, the modified fixed point equation based on (3.8) reads as

$$
\phi^{j+1}=\mathcal{R}^{-1} \circ \widetilde{\mathcal{T}}\left[\phi^{j}\right]
$$

for $j \in \mathbb{N}$.

We use different discrete ansatz spaces for the deformations and the images. Furthermore, we choose the conforming space of cubic splines $\mathcal{S}_{H} \subset C^{2}(\Omega)$ as the discrete ansatz space for deformations. Here $H=2^{-N}$ with $N \in \mathbb{N}$ denotes the grid size of the underlying uniform and rectangular mesh, and the basis functions are vector-valued B-splines. Moreover, we only impose the Dirichlet boundary condition $\boldsymbol{\Phi}=\mathbb{1}$ on $\partial \Omega$ instead of the stronger boundary condition $\boldsymbol{\Phi}-\mathbb{1} \in H_{0}^{2}(\Omega)$ for the discrete deformations $\boldsymbol{\Phi} \in \mathcal{S}_{H}$. Indeed, we experimentally observed that this Dirichlet boundary condition allows us to reliably compute proper deformations. The gray value images are approximated with finite element functions in the space $\mathcal{V}_{h}$ of piecewise bilinear and globally continuous functions on $\Omega$ with input intensities in the range $[0,1]$. The underlying grid consists of uniform and quadratic cells with mesh size $h=2^{-M}$ with $M>N$; the index set of all grid nodes is denoted by $I_{\mathcal{V}_{h}}^{N}$. We take into account the usual Lagrange basis functions $\left\{\Theta^{i}\right\}_{i \in I_{\mathcal{V}_{h}}^{N}}$ to represent image intensities $\mathbf{U} \in \mathcal{V}_{h}$. In our numerical experiments we set $M=N+1$.

Now we are in the position to define spatially discrete counterparts of the energy and the operators involved in the fixed point iteration. We apply a Gaussian quadrature of order 5 on both meshes. The discrete energy for $\mathbf{U}, \tilde{\mathbf{U}} \in \mathcal{V}_{h}$ and $\mathbf{\Phi} \in \mathcal{S}_{H}$ is defined as (cf. (2.8))

$$
\begin{aligned}
\mathbf{W}^{D}[\mathbf{U}, \tilde{\mathbf{U}}, \boldsymbol{\Phi}]= & \sum_{c_{H}} \sum_{q_{H}} \omega_{q_{H}}^{c_{H}}\left((D \boldsymbol{\Phi}-\mathbb{1})\left(\mathbf{x}_{q_{H}}^{c_{H}}\right):(D \boldsymbol{\Phi}-\mathbb{1})\left(\mathbf{x}_{q_{H}}^{c_{H}}\right)+\gamma \Delta \boldsymbol{\Phi}\left(\mathbf{x}_{q_{H}}^{c_{H}}\right) \cdot \Delta \boldsymbol{\Phi}\left(\mathbf{x}_{q_{H}}^{c_{H}}\right)\right) \\
& +\frac{1}{\delta} \sum_{c_{h}} \sum_{q_{h}} \omega_{q_{h}}^{c_{h}}\left(\tilde{\mathbf{U}}\left(\boldsymbol{\Phi}\left(\mathbf{x}_{q_{h}}^{c_{h}}\right)\right)-\mathbf{U}\left(\mathbf{x}_{q_{h}}^{c_{h}}\right)\right)^{2},
\end{aligned}
$$

where we sum over all grid cells $c_{H}$ of the spline mesh and all local quadrature points within these cells indexed by $q_{H}$ w.r.t. the deformation energy and over all grid cells $c_{h}$ of the finer finite element mesh and all local quadrature points within these cells indexed by $q_{h}$. Here $\left(\omega_{q_{H}}^{c_{H}}, \mathbf{x}_{q_{H}}^{c_{H}}\right)$ and $\left(\omega_{q_{h}}^{c_{h}}, \mathbf{x}_{q_{h}}^{c_{h}}\right)$ are the pairs of quadrature weights and points on the spline mesh and the finite element mesh, respectively. For the fully discrete counterparts of the operators $\widetilde{\mathcal{T}}$ and $\mathcal{R}$ one gets

$$
\widetilde{\mathbf{T}}[\boldsymbol{\Phi}](\boldsymbol{\Psi})=\sum_{c_{H}} \sum_{q_{H}} \omega_{q_{H}}^{c_{H}}\left(-2 \gamma\left((D \boldsymbol{\Phi})^{-T} \circ \boldsymbol{\Phi}_{1}\left(\mathbf{x}_{q_{H}}^{c_{H}}\right)\right) D \Delta \boldsymbol{\Phi}_{1}\left(\mathbf{x}_{q_{H}}^{c_{H}}\right): D\left(\boldsymbol{\Psi} \circ \boldsymbol{\Phi}_{1}\left(\mathbf{x}_{q_{H}}^{c_{H}}\right)\right)\right.
$$




$$
\begin{gathered}
-2 \gamma D \Delta \boldsymbol{\Phi}_{1}\left(\mathbf{x}_{q_{H}}^{c_{H}}\right): D\left((D \boldsymbol{\Phi})^{-1} \circ \mathbf{\Phi}_{1}\left(\mathbf{x}_{q_{H}}^{c_{H}}\right)\right)\left(\boldsymbol{\Psi} \circ \boldsymbol{\Phi}_{1}\left(\mathbf{x}_{q_{H}}^{c_{H}}\right)\right) \\
\left.-2 \Delta \boldsymbol{\Phi}_{1}\left(\mathbf{x}_{q_{H}}^{c_{H}}\right) \cdot\left((D \boldsymbol{\Phi})^{-1} \mathbf{\Psi}\right) \circ \boldsymbol{\Phi}_{1}\left(\mathbf{x}_{q_{H}}^{c_{H}}\right)\right) \\
-\sum_{c_{h}} \sum_{q_{h}} \frac{\omega_{q_{h}}^{c_{h}}}{\delta} \frac{\left(\mathbf{U}_{1} \circ \boldsymbol{\Phi}_{1}\left(\mathbf{x}_{q_{h}}^{c_{h}}\right)-\mathbf{U}_{0}\left(\mathbf{x}_{q_{h}}^{c_{h}}\right)\right)^{2}}{\operatorname{det} D \boldsymbol{\Phi}_{1}\left(\mathbf{x}_{q_{h}}^{c_{h}}\right)} \\
\cdot\left((D \boldsymbol{\Phi})^{-T}:\left(D^{2} \mathbf{\Phi}(D \boldsymbol{\Phi})^{-1} \mathbf{\Psi}\right)-(D \boldsymbol{\Phi})^{-T}: D \boldsymbol{\Psi}\right) \circ \boldsymbol{\Phi}_{1}\left(\mathbf{x}_{q_{h}}^{c_{h}}\right), \\
\mathbf{R}[\boldsymbol{\Phi}](\boldsymbol{\Psi})=\sum_{c_{H}} \sum_{q_{H}} \omega_{q_{H}}^{c_{H}}\left(2 \gamma \Delta \boldsymbol{\Phi}\left(\mathbf{x}_{q_{H}}^{c_{H}}\right) \cdot \Delta \boldsymbol{\Psi}\left(\mathbf{x}_{q_{H}}^{c_{H}}\right)+2 D \boldsymbol{\Phi}\left(\mathbf{x}_{q_{H}}^{c_{H}}\right): D \boldsymbol{\Psi}\left(\mathbf{x}_{q_{H}}^{c_{H}}\right)\right)
\end{gathered}
$$

for all $\boldsymbol{\Psi} \in \mathcal{S}_{H}$ with $\boldsymbol{\Psi}=0$ on $\partial \Omega$. Finally, one obtains the following fixed point iteration to compute the spatially discrete deformation $\boldsymbol{\Phi}_{2}$ :

$$
\boldsymbol{\Phi}^{j+1}=\mathbf{R}^{-1} \circ \widetilde{\mathbf{T}}\left[\Phi^{j}\right]
$$

for all $j \geq 0$ and initial data $\boldsymbol{\Phi}^{0}=\mathbb{1}$. The application of $\mathbf{R}^{-1}$ requires the solution of the associated linear system of equations.

In a preparatory step, the deformation $\boldsymbol{\Phi}_{1} \in \operatorname{argmin}_{\boldsymbol{\Phi} \in \mathcal{S}_{H}} \mathbf{W}^{D}\left[\mathbf{U}_{0}, \mathbf{U}_{1}, \boldsymbol{\Phi}\right]$, which is used in the first step of a time discrete geodesic shooting, is calculated using a Fletcher-Reeves nonlinear conjugate gradient descent multilevel scheme with an Armijo step size control.

Then the deformation in the current step is computed using the fixed point iteration (5.1), which is stopped if the $L^{\infty}$-difference of the deformations in two consecutive iterations is below the threshold value THRESHOLD $=10^{-12}$. To compute $\mathbf{U}_{2}$ we employ the spatially discrete analogue of the update formula (3.11):

$$
\mathbf{U}_{2}(\mathbf{x})=\left(\frac{\mathbf{U}_{1}-\mathbf{U}_{0} \circ \boldsymbol{\Phi}_{1}^{-1}}{\operatorname{det}\left(D \boldsymbol{\Phi}_{1}\right) \circ \boldsymbol{\Phi}_{1}^{-1}}\right) \circ \boldsymbol{\Phi}_{2}^{-1}(\mathbf{x})+\mathbf{U}_{1} \circ \boldsymbol{\Phi}_{2}^{-1}(\mathbf{x}) .
$$

Here we evaluate (5.2) at all grid nodes of the finite element grid. To compute approximate inverse deformations $\boldsymbol{\Phi}_{i}^{-1} \in \mathcal{S}_{H}, i \in\{1,2\}$, all cells of the grid associated with $\mathcal{S}_{H}$ are traversed and the deformed positions $\boldsymbol{\Phi}_{i}\left(\mathbf{x}_{j}\right)$ for all vertices $\mathbf{x}_{j}, j \in\{1, \ldots, 4\}$, of the current element are computed. Then we use a bilinear interpolation of these deformed positions to define an approximation of $\boldsymbol{\Phi}_{i}^{-1}(x)$ for $x \in \Omega$. Furthermore, we explicitly ensure the boundary condition $\boldsymbol{\Phi}_{i}^{-1}(x)=x$ for $x \in \partial \Omega$.

In our numerical experiments on real image data we observed slight local oscillations emerging from the inexact evaluation of the expression $\mathbf{J}_{k}=\mathbf{U}_{k}(\mathbf{x})-\mathbf{U}_{k-1} \circ \boldsymbol{\Phi}_{k}^{-1}(\mathbf{x})$ in the quadrature of the intensity modulation. Since the calculation of $\mathrm{EXP}^{k}$ requires a recursive application of $\mathrm{EXP}^{2}$, these oscillations turn out to be sensitive to error propagation, and it is advantageous to apply in a postprocessing step one iteration of the anisotropic diffusion filter $\left(\mathbf{M}_{h}+\tau \mathbf{S}_{h}\left[\mathbf{J}_{k}, \lambda\right]\right)^{-1} \mathbf{M}_{h}$ to $\mathbf{J}_{k}$ for weight parameters $\tau, \lambda>0$ (see [27]). Here $\mathbf{M}_{h}$ is the usual mass matrix and $\mathbf{S}_{h}\left[\mathbf{J}_{k}, \lambda\right]$ the anisotropic stiffness matrix associated with $\mathcal{V}_{h}$, i.e.,

$$
\left(\mathbf{S}_{h}\left[\mathbf{J}_{k}, \lambda\right]\right)_{i, j}=\sum_{c_{h}, q_{h}} \omega_{q_{h}}^{c_{h}}\left(1+\lambda^{-2}\left\|\nabla \mathbf{J}_{k}\left(\mathbf{x}_{q_{h}}^{c_{h}}\right)\right\|^{2}\right)^{-1} \nabla \Theta^{i}\left(\mathbf{x}_{q_{h}}^{c_{h}}\right) \cdot \nabla \Theta^{j}\left(\mathbf{x}_{q_{h}}^{c_{h}}\right)
$$


for $i, j \in I_{\mathcal{V}_{h}}^{N}$. Furthermore, in all the following applications except the first test case (Figure 2) we choose $\tau=\beta^{k-2} \cdot 10^{-3}$ as the exponentially decaying time step size ( $k$ denoting the index of the image in the sequence and $\beta=0.8)$ and $\lambda=0.5$ as the smoothing parameter along the discrete geodesic. The impact of this filtering can be seen, for instance, in Figure 3.
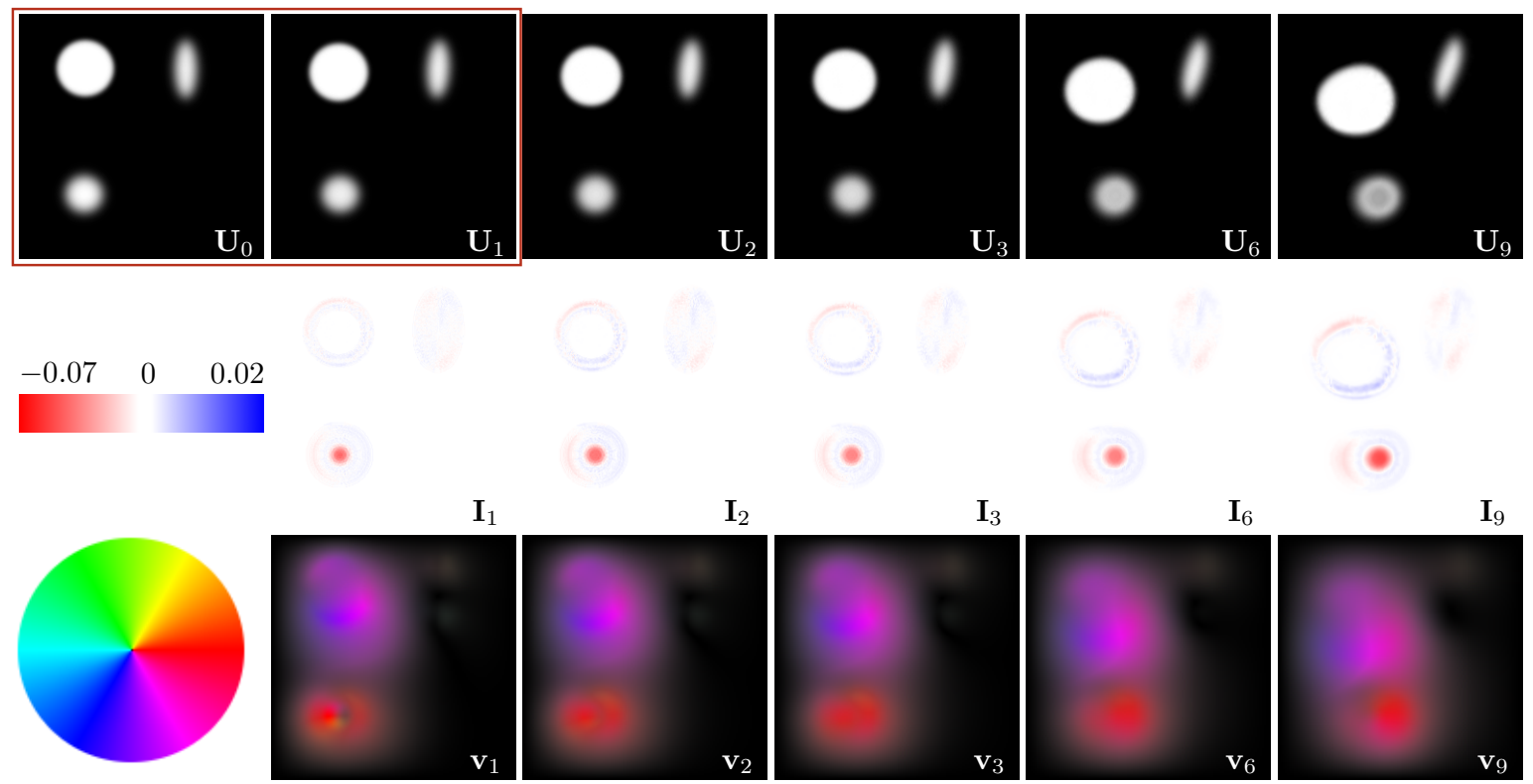

Figure 2. First row: the discrete exponential map $\operatorname{EXP}_{\mathbf{U}_{0}}^{k}\left(\mathbf{U}_{1}-\mathbf{U}_{0}\right)$ with $k=0,1,2,3,6,9$ for images showing three ellipses (input images are framed in red). Second row: the associated intensity modulations $\mathbf{I}_{k}$. Third row: the discrete velocity fields $\mathbf{v}_{k}$ (the hue refers to the direction; the intensity is proportional to its norm).

6. Numerical results. In this section, we present applications of the fully discrete exponential map. In all computations, we use the parameters $\gamma=10^{-4}$ and $\delta=10^{-2}$.

As a first example, we investigate an artificial test case consisting of an input image $\mathbf{U}_{0}$ with three ellipses of different intensities and an associated variation $\mathbf{U}_{1}-\mathbf{U}_{0}$. The first row in Figure 2 depicts distinct images of the image sequence $\operatorname{EXP}_{\mathbf{U}_{0}}^{k}\left(\mathbf{U}_{1}-\mathbf{U}_{0}\right)$ for time steps $k=0,1,2,3,6,9$; the input images $\mathbf{U}_{0}$ and $\mathbf{U}_{1}$ with resolution $257 \times 257$ are framed in red. In the initial variation $\mathbf{U}_{1}-\mathbf{U}_{0}$ underlying the exponential shooting, the upper left ellipse is slightly translated to the bottom and simultaneously expanded. The upper right ellipse is undergoing a small rotation, and the third one is also slightly translated with some modulation of the shading. The initial variation encoded in the image pair $\left(\mathbf{U}_{0}, \mathbf{U}_{1}\right)$ is prolongated along the sequence generated by an iterative application of the discrete exponential map EXP2. In the second row, for each $k>0$ the discrete intensity modulations $\mathbf{I}_{k}=\mathbf{U}_{k} \circ \boldsymbol{\Phi}_{k}-\mathbf{U}_{k-1}$ are visualized. Here, on the left, the color bar with bounds coinciding with the extremal values for this image sequence is displayed. The third row depicts the discrete velocity fields $\mathbf{v}_{k}=\frac{1}{\tau}\left(\Phi_{k}-\mathbb{1}\right)$ for each $k$, where $\tau=\frac{1}{K}$ is the associated time step size. Here the hue refers 

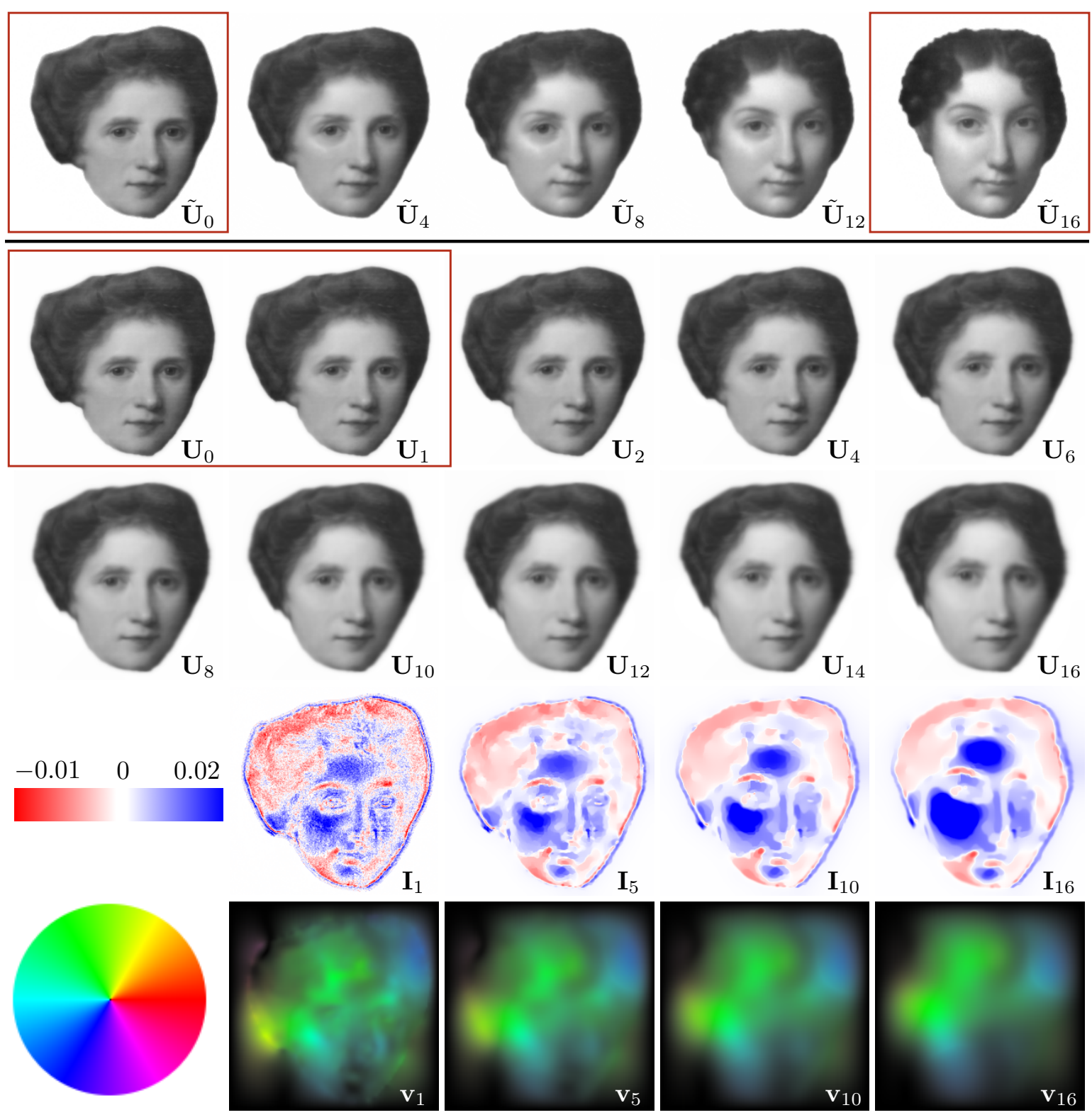

Figure 3. The first row depicts distinct images of the discrete geodesic sequence associated with the input images $\tilde{\mathbf{U}}_{0}$ and $\tilde{\mathbf{U}}_{16}$ (in red boxes). The discrete exponential map for distinct time steps $k$ is shown in the second and third rows, where the input images $\mathbf{U}_{0}$ and $\mathbf{U}_{1}$ coincide with $\tilde{\mathbf{U}}_{0}$ and $\tilde{\mathbf{U}}_{1}$ from the geodesic sequence, respectively. In addition, the corresponding intensity modulations as well as the discrete velocity fields (fourth and fifth rows) are shown for some time steps $k$.

to the direction and the color intensity is proportional to the local norm of $\mathbf{v}_{k}$ as represented by the leftmost color wheel. In particular, one observes that the resulting underlying velocity field $\mathbf{v}_{k}$ is not constant in time. 
In [6, Figure 6.2], a geodesic sequence between two female portrait paintings ${ }^{1}$ was computed using the finite element discretization for both the images and the deformations on the same grid. The image resolution is $257 \times 257(M=8)$. We recomputed this geodesic sequence $\left(\tilde{\mathbf{U}}_{0}, \tilde{\mathbf{U}}_{1}, \ldots, \tilde{\mathbf{U}}_{16}\right)$ with $K=16$ for the discrete function spaces $\mathcal{V}_{h}$ and $\mathcal{S}_{H}$ with $N=7$; the resulting sequence is shown in the first row of Figure 3 with framed input images $\tilde{\mathbf{U}}_{0}$ and $\tilde{\mathbf{U}}_{16}$. This is compared with the discrete exponential shooting for the initial image pair $\left(\mathbf{U}_{0}, \mathbf{U}_{1}\right)$ taken from this geodesic sequence. One observes that the discrete exponential map is capable of recovering the original geodesic sequence for small time steps $k$. Only in late stages do visible differences become apparent. Again, we highlight that the discrete motion fields significantly alter in time.

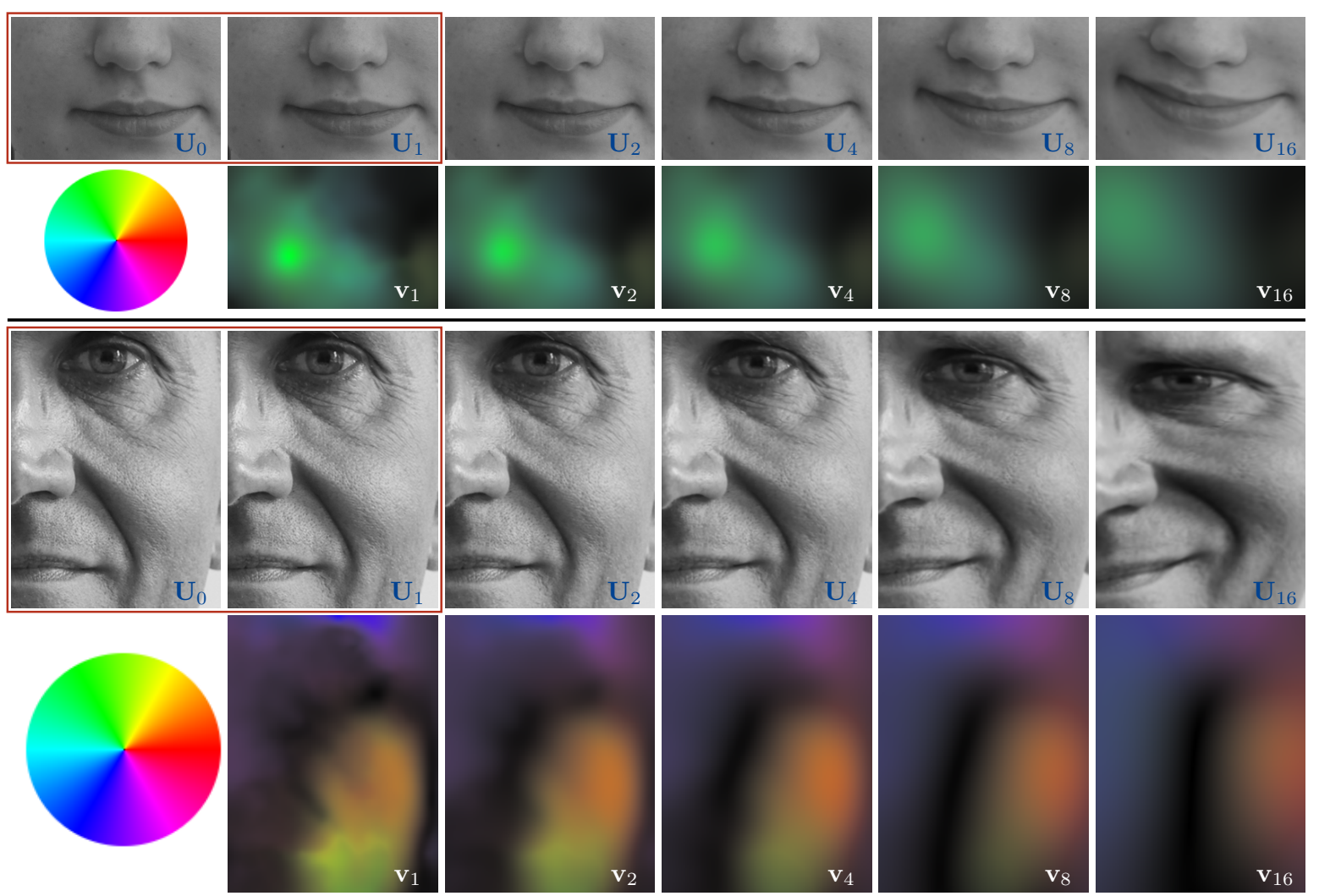

Figure 4. First/third rows: picture details of $\operatorname{EXP}_{\mathbf{U}_{0}}^{k}\left(\mathbf{U}_{1}-\mathbf{U}_{0}\right)$ applied to two pairs of photos of human faces for time steps $k=0,1,2,4,8,16$. Second/fourth rows: the associated discrete velocity fields $\mathbf{v}_{k}$.

Figure 4 depicts picture details for the time steps $k=0,1,2,4,8,16$ of the discrete exponential map applied to two different pairs of photos. These photos show human faces and small variations of them, and the resolution of the underlying full images is $1025 \times 1025$. The red boxes indicate these input images (first and third rows), which are consecutive photos of

\footnotetext{
${ }^{1}$ First painting by A. Kauffmann (public domain; see http://commons.wikimedia.org/wiki/File:Angelika Kauffmann_-_Self_Portrait_-_1784.jpg), second painting by R. Peale (GFDL; see http://en.wikipedia.org/wiki/ File:Mary_Denison.jpg).
} 
a series at 5 and $7 \mathrm{fps}$, respectively, taken with a digital camera. We observe that small initial variations result in a nonlinear deformation of the lips (first row) and of the lips, the cheeks, and the eyes (third row), respectively. Furthermore, the textures are transported along the sequence. The second and fourth rows depict the color-coded time-varying velocity fields.
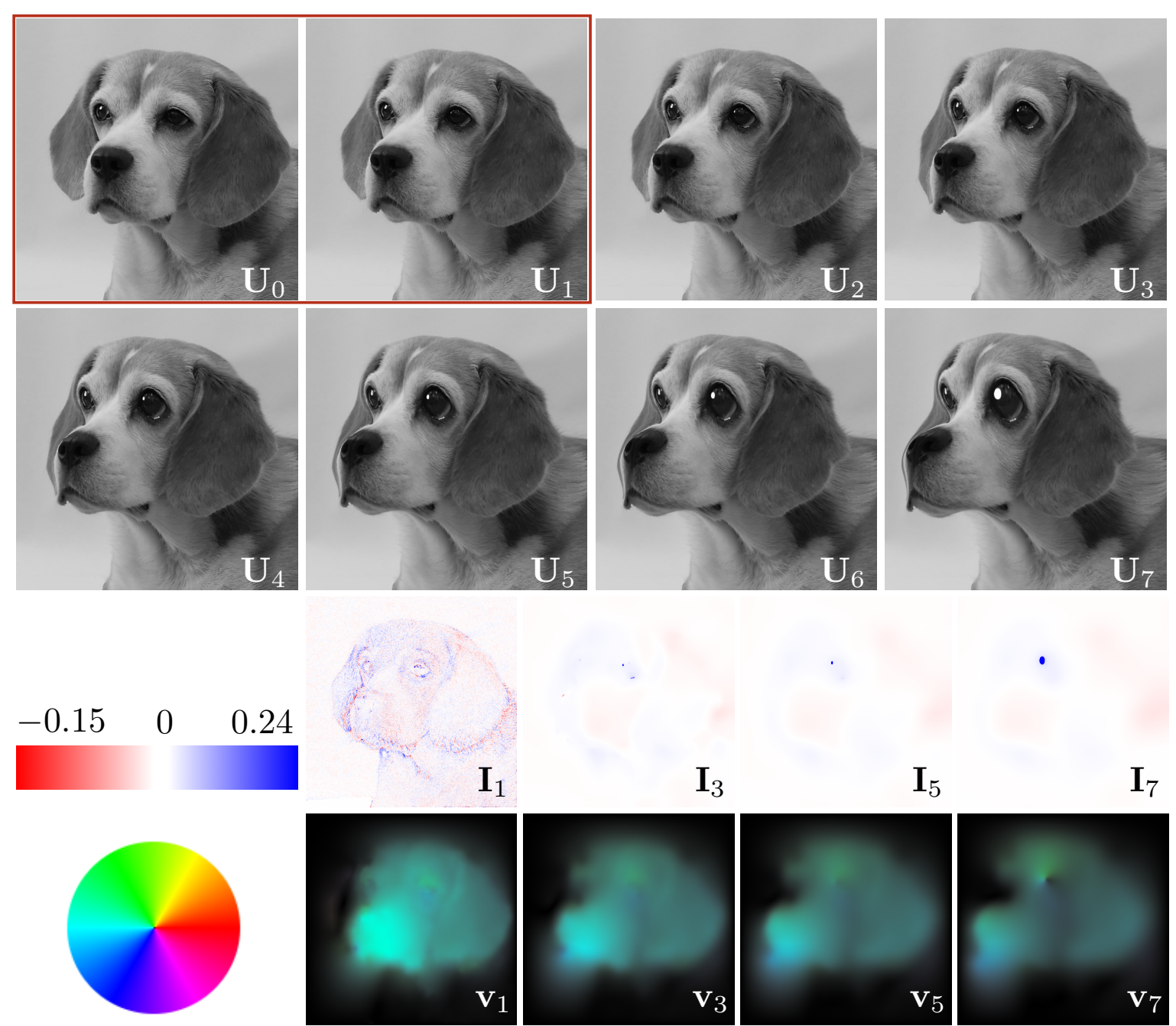

Figure 5. The discrete exponential map for time steps $k=0, \ldots, 7$ with two photos of a dog as initial data (first and second rows). Third/fourth rows: the associated intensity modulations and velocity fields for distinct time steps.

Figure 5 shows the discrete exponential map for $k=0, \ldots, 7$ applied to a pair of images of a dog for a resolution of $1025 \times 1025$. Again, the input pictures are consecutive photos of a series with $7 \mathrm{fps}$ taken with a digital camera. The initial image pair shows a slight rotation of the dog's head and a small opening of its eyes. The proposed algorithm generates an extrapolation of this movement. As a consequence, in particular of the rotation, the method fills in reasonable image features below the mouth and right of the ear which correspond to 
hidden object regions in $\mathbf{U}_{0}$ and $\mathbf{U}_{1}$.

The computational complexity of the method for increasing image resolution is asymptotically dominated by the solution of the fourth order linear system (5.1). The condition number of this system is $\mathcal{O}\left(\gamma h^{-4}\right)$ for the grid size $h$, and thus the complexity of the conjugate gradient solver scales quadratically in the image resolution. In fact, for moderate image resolutions up to $1000^{2}$, the computational bottleneck is the compilation of the nonlinear terms of the discretized fixed point problem via higher order quadrature rules, where the complexity scales only linearly with the degrees of freedom.

Altogether, the computation time of the algorithm depends on the length of the extrapolated path, the size of the image, and the chosen stopping value in the fixed point iteration for the $L^{\infty}$-distance of consecutive deformations. For instance, the total computation time for the "woman sequence" (size $257 \times 257, K=16$, very small threshold value $10^{-12}$ for the $L^{\infty}$-distance) was about 14 minutes (including the initial registration) when using four parallel threads (Intel(R) Core(TM) i7-2600 CPU 3.40GHz).

Supplementary material. The supplementary material for this paper (M112954_01.mp4 [local/web 6.46MB]), which is linked from the main article webpage, includes video sequences with animations of the discrete exponential maps shown in Figures 3 (together with the discrete geodesic interpolation), 4, and 5 .

\section{REFERENCES}

[1] R. A. Adams and J. J. F. Fournier, Sobolev Spaces, 2nd ed., Pure. Appl. Math. (Amsterdam) 140, Elsevier/Academic Press, Amsterdam, 2003, https://www.elsevier.com/books/sobolev-spaces/adams/ 978-0-12-044143-3 (accessed 2018-01-16).

[2] V. ARnold, Sur la géométrie différentielle des groupes de Lie de dimension infinie et ses applications à l'hydrodynamique des fluides parfaits, Ann. Inst. Fourier (Grenoble), 16 (1966), pp. 319-361, https: //doi.org/10.5802/aif.233.

[3] V. Arnold and B. Khesin, Topological Methods in Hydrodynamics, Springer, New York, 1998, https: //doi.org/10.1007/B97593.

[4] M. F. Beg, M. I. Miller, A. Trouvé, And L. Younes, Computing large deformation metric mappings via geodesic flows of diffeomorphisms, Int. J. Comput. Vis., 61 (2005), pp. 139-157, https://doi.org/ 10.1023/B:VISI.0000043755.93987.aa.

[5] B. Berkels, M. Buchner, A. Effland, M. Rumpf, and S. Schmitz-Valckenberg, GPU-based image geodesics for optical coherence tomography, in Bildverarbeitung für die Medizin, Informatik aktuell, Springer, Berlin, Heidelberg, 2017, pp. 68-73, https://doi.org/10.1007/978-3-662-54345-0_21.

[6] B. Berkels, A. Effland, AND M. Rumpf, Time discrete geodesic paths in the space of images, SIAM J. Imaging Sci., 8 (2015), pp. 1457-1488, https://doi.org/10.1137/140970719.

[7] P. G. Ciarlet, Mathematical Elasticity. Volume I: Three-Dimensional Elasticity, Stud. Math. Appl. 20, North-Holland, Amsterdam, 1988, https://www.elsevier.com/books/three-dimensional-elasticity/ ciarlet/978-0-444-70259-3 (accessed 2018-01-16).

[8] P. Dupuis, U. Grenander, And M. I. Miller, Variational problems on flows of diffeomorphisms for image matching, Quart. Appl. Math., 56 (1998), pp. 587-600, https://doi.org/10.1090/qam/1632326.

[9] A. Effland, M. Rumpf, AND F. SchäFer, Time discrete extrapolation in a Riemannian space of images, in Proceedings of the International Conference on Scale Space and Variational Methods in Computer Vision, Lecture Notes in Comput. Sci. 10302, Springer, Cham, 2017, pp. 473-485, https://doi.org/10.1007/978-3-319-58771-4_38.

[10] A. Effland, M. Rumpf, S. Simon, K. Stahn, and B. Wirth, Bézier curves in the space of images, in Proceedings of the International Conference on Scale Space and Variational Methods in Computer

Copyright ( $)$ by SIAM. Unauthorized reproduction of this article is prohibited. 
Vision, Lecture Notes in Comput. Sci. 9087, Springer, Cham, 2015, pp. 372-384, https://doi.org/10. 1007/978-3-319-18461-6_30.

[11] F. Gazzola, H.-C. Grunau, and G. Sweers, Polyharmonic Boundary Value Problems. Positivity Preserving and Nonlinear Higher Order Elliptic Equations in Bounded Domains, Lecture Notes in Math. 1991, Springer-Verlag, Berlin, 2010, https://doi.org/10.1007/978-3-642-12245-3.

[12] D. Gilbarg and N. S. Trudinger, Elliptic Partial Differential Equations of Second Order, Classics Math., Springer-Verlag, Berlin, 1992, https://doi.org/10.1007/978-3-642-61798-0.

[13] A. E. Green And R. S. Rivlin, Multipolar continuum mechanics, Arch. Rational Mech. Anal., 17 (1964), pp. 113-147, https://doi.org/10.1007/bf00253051.

[14] A. E. Green And R. S. Rivlin, Simple force and stress multipoles, Arch. Rational Mech. Anal., 16 (1964), pp. 325-353, https://doi.org/10.1007/978-1-4612-2416-7_113.

[15] G. L. Hart, C. ZACH, AND M. Niethammer, An optimal control approach for deformable registration, in Proceedings of the IEEE Computer Society Conference on Computer Vision and Pattern Recognition, 2009, https://doi.org/10.1109/CVPRW.2009.5204344.

[16] D. Holm, A. Trouvé, And L. Younes, The Euler-Poincaré theory of metamorphosis, Quart. Appl. Math., 67 (2009), pp. 661-685, https://doi.org/10.1090/S0033-569X-09-01134-2.

[17] Y. Hong, S. Joshi, M. SAnchez, M. Styner, And M. Niethammer, Metamorphic geodesic regression, in Proceedings of the International Conference on Medical Image Computing and Computer-Assisted Intervention, Lecture Notes in Comput. Sci. 7512, Springer, Berlin, Heidelberg, 2012, pp. 197-205, https://doi.org/10.1007/978-3-642-33454-2_25.

[18] S. C. Joshi And M. I. Miller, Landmark matching via large deformation diffeomorphisms, IEEE Trans. Image Process., 9 (2000), pp. 1357-1370, https://doi.org/10.1109/83.855431.

[19] W. P. A. Klingenberg, Riemannian Geometry, 2nd ed., De Gruyter Stud. Math. 1, Walter de Gruyter \& Co., Berlin, 1995, https://doi.org/10.1515/9783110905120.

[20] M. Lorenzi And X. Pennec, Geodesics, parallel transport 63 one-parameter subgroups for diffeomorphic image registration, Int. J. Comput. Vis., 105 (2013), pp. 111-127, https://doi.org/10.1007/ s11263-012-0598-4.

[21] J. E. Marsden And M. West, Discrete mechanics and variational integrators, Acta Numer., 10 (2001), pp. 357-514, https://doi.org/10.1017/S096249290100006X.

[22] M. I. Miller, A. Trouvé, And L. Younes, On the metrics and Euler-Lagrange equations of computational anatomy, Annu. Rev. Biomed. Eng., 4 (2002), pp. 375-405, https://doi.org/10.1146/annurev. bioeng.4.092101.125733.

[23] M. I. Miller, A. Trouvé, And L. Younes, Geodesic shooting for computational anatomy, J. Math. Imaging Vision, 24 (2006), pp. 209-228, https://doi.org/10.1007/s10851-005-3624-0.

[24] M. I. Miller And L. Younes, Group actions, homeomorphisms, and matching: A general framework, Int. J. Comput. Vis., 41 (2001), pp. 61-84, https://doi.org/10.1023/A:1011161132514.

[25] J. NeČas And M. ŠılhavÝ, Multipolar viscous fluids, Quart. Appl. Math., 49 (1991), pp. 247-265, https://doi.org/10.1090/qam/1106391.

[26] S. Ober-Blöbaum, O. Junge, and J. E. Marsden, Discrete mechanics and optimal control: An analysis, ESAIM Control Optim. Calc. Var., 17 (2011), pp. 322-352, https://doi.org/10.1051/cocv/ 2010012.

[27] P. Perona AND J. MALIK, Scale-space and edge detection using anisotropic diffusion, IEEE Trans. Pattern Anal. Mach. Intell., 12 (1990), pp. 629-639, https://doi.org/10.1109/34.56205.

[28] M. Rumpf And B. Wirth, Variational time discretization of geodesic calculus, IMA J. Numer. Anal., 35 (2015), pp. 1011-1046, https://doi.org/10.1093/imanum/dru027.

[29] A. Trouvé, An Infinite Dimensional Group Approach for Physics Based Models in Pattern Recognition, 1995, http://www.cis.jhu.edu/publications/papers_in_database/alain/trouve1995.pdf (accessed 201801-16).

[30] A. Trouvé, Diffeomorphisms groups and pattern matching in image analysis, Int. J. Comput. Vis., 28 (1998), pp. 213-221, https://doi.org/10.1023/A:1008001603737.

[31] A. Trouvé and L. Younes, Local geometry of deformable templates, SIAM J. Math. Anal., 37 (2005), pp. 17-59, https://doi.org/10.1137/S0036141002404838.

[32] A. Trouvé And L. Younes, Metamorphoses through Lie group action, Found. Comput. Math., 5 (2005), pp. 173-198, https://doi.org/10.1007/s10208-004-0128-z.

Copyright (C) by SIAM. Unauthorized reproduction of this article is prohibited. 
[33] F.-X. Vialard, L. Risser, D. Rueckert, And C. J. Cotter, Diffeomorphic 3D image registration via geodesic shooting using an efficient adjoint calculation, Int. J. Comput. Vis., 97 (2012), pp. 229-241, https://doi.org/10.1007/s11263-011-0481-8.

[34] F.-X. Vialard, L. Risser, D. Rueckert, and D. D. Holm, Diffeomorphic atlas estimation using geodesic shooting on volumetric images, Ann. BMVA, 2012 (2012), pp. 1-12, http://www.bmva.org/ annals/2012/2012-0005.pdf (accessed 2018-01-16).

[35] F.-X. Vialard and F. Santambrogio, Extension to $B V$ functions of the large deformation diffeomorphisms matching approach, C. R. Math. Acad. Sci. Paris, 347 (2009), pp. 27-32, https: //doi.org/10.1016/j.crma.2008.11.019.

[36] L. Younes, Jacobi fields in groups of diffeomorphisms and applications, Quart. Appl. Math, 65 (2007), pp. 113-134, https://doi.org/10.1090/S0033-569X-07-01027-5.

[37] L. Younes, Shapes and Diffeomorphisms, Appl. Math. Sci. 171, Springer-Verlag, Berlin, 2010, https: //doi.org/10.1007/978-3-642-12055-8.

Copyright $\odot$ by SIAM. Unauthorized reproduction of this article is prohibited. 